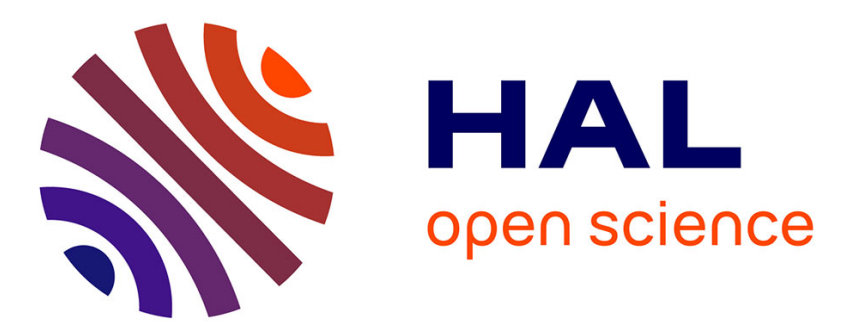

\title{
Elasto-geometrical modeling and calibration of robot manipulators: Application to machining and forming applications
}

Stéphane Marie, Eric Courteille, Patrick Maurine

\section{To cite this version:}

Stéphane Marie, Eric Courteille, Patrick Maurine. Elasto-geometrical modeling and calibration of robot manipulators: Application to machining and forming applications. Mechanism and Machine Theory, 2013, 69, pp.13-43. 10.1016/j.mechmachtheory.2013.05.00 . hal-00917604

\author{
HAL Id: hal-00917604 \\ https://hal.science/hal-00917604
}

Submitted on 12 Dec 2013

HAL is a multi-disciplinary open access archive for the deposit and dissemination of scientific research documents, whether they are published or not. The documents may come from teaching and research institutions in France or abroad, or from public or private research centers.
L'archive ouverte pluridisciplinaire HAL, est destinée au dépôt et à la diffusion de documents scientifiques de niveau recherche, publiés ou non, émanant des établissements d'enseignement et de recherche français ou étrangers, des laboratoires publics ou privés. 


\title{
Elasto-geometrical modeling and calibration of robot manipulators: Application to machining and forming applications
}

\author{
Stéphane MARIE ${ }^{a, *}$, Eric COURTEILLE ${ }^{a}$, Patrick MAURINE ${ }^{a}$ \\ ${ }^{a}$ Université Européenne de Bretagne, INSA-LGCGM - EA 3913
}

\begin{abstract}
This paper proposes an original elasto-geometrical calibration method to improve the static pose accuracy of industrial robots involved in machining, forming or assembly applications. Two approaches are presented respectively based on an analytical parametric modeling and a Takagi-Sugeno fuzzy inference system. These are described and then discussed. This allows to list the main drawbacks and advantages of each of them with respect to the task and the user requirements. The Fuzzy Logic model is used in a model-based compensation scheme to increase significantly the robot static pose accuracy in a context of incremental forming application. Experimental results show the efficiency of the Fuzzy Logic model while minimizing development and computational resources.
\end{abstract}

Keywords: Elasto-geometrical modeling, robot calibration, stiffness identification, Fuzzy Logic modeling, data-driven identification, forming applications

\section{Introduction}

Industrial robots propose an interesting alternative to dedicated machines, when it comes to perform applications such as machining, forming or assembly since they offer the advantage of a very large working envelope compared to traditional machine tools or machining centers $[1,2]$. However these applications require that the robots apply high level force at the TCP but their serial open structures lack stiffness. As a result consequent elastic deformations of the robot generate TCP pose errors that degrade the process in terms of geometry, surface state, etc $[3,4,5,6,7]$. Therefore rigidity and accuracy remain the major obstacles to the widespread use of robots for these processes. In order to overcome these problems different solutions are available.

The first solution consists in modifying their structure with shortened links, increased sections, parallelogram loops or increased reduction gear ratios and actuator powers. This kind of robots are already available such as the KUKA

\footnotetext{
* Corresponding author at : 20, avenue des Buttes de Coesmes 35043 RENNES Cedex, France. Tel.: + 336365090 93; fax: +33223238726

Email addresses: stephane.marie61@gmail.com (Stéphane MARIE), eric.courteille@insa-rennes.fr (Eric COURTEILLE), patrick.maurine@insa-rennes.fr (Patrick MAURINE)
} 
500-2 MT or the ABB IR7600-500 but these machines remain less versatile and more expensive than usual structures.

The second solution, which is more economical, is to perform the identification of both geometrical and elastic models of the robot's structure or, in other words, to perform an elasto-geometrical calibration. In past decades, much of the work in the area of robot calibration has concerned geometrical calibration including studies about the modeling of their structure $[8,9,10,11]$, the measurement data collection [12,13] and the error model identification [14]. Due to the difficulty to modify the model's parameters within the robot controller, the proposed methods use fakes poses to enhance the position and the orientation of the tool. Compared to geometrical calibration, only few works have investigated the compensation of the TCP pose errors due to elastic deformations. For that purpose two main approaches are available in the literature.

Some authors proposed dynamic models of the robot's structure in order to compensate the pose errors due to elastic effects by linear or non-linear feedback controls $[15,16,17]$. In these methods, the actuator torques are modified by the control and are therefore difficult to implement on actual industrial robots. Even if open controller would be ideal, actual robot controllers only allow the TCP position/orientation to be controlled. Moreover, the dynamic parameters (inertia, center of gravity, gear ratio) must be identified by dedicated methodologies $[18,19,20]$.

The other approach consists in deriving realistic parametric models to predict the elastic deformations. In the literature, the proposed methods are:

- Lumped-parameter model: These models describe the structure of a manipulators as a set of rigid bodies, which are connected by springs [3, 4, $13,21,22,23,24,25,26,27,28,29]$. These springs are used to describe the elastic behavior of the joints and the links. It has the advantage to be easily derived but its accuracy and consistency depend on the number, size and location of the springs used.

- Finite element model:

- The structure's elastic displacements can be calculated by assuming the robot's links as beam elements according the Euler-Bernoulli's theory. This allows to calculate the parametric equivalent stiffness matrix of the structure from which the elastic model is derived in a systematic and analytical way $[30,31,32]$. This kind of method is used here for the calculation of the TCP pose errors resulting from the structure's elastic displacements.

- The elastic displacements can also be obtained with volumetric FE CAD softwares but the calculation time does not allow its use for quick estimation and for real time pose or trajectory compensations [33, 34, 35].

These traditional physical modeling methods assume a complete knowledge and understanding of the physical model interrelationships. However some effects cannot be easily integrated in the model as the non-linear joint's stiffness with respect to robot joint configurations for instance. In order to overcome this problem, a fuzzy modeling approach for data-driven identification of the elastic behavior of robot manipulators can be used [36, 37, 38, 39, 40]. The main 
advantage of this approach is to quickly develop an accurate model without assumptions about the structure's elasto-geometrical behavior. The resulting fuzzy inference system can accurately describe the elastic behavior while minimizing development and computational requirements.

The work presented in this paper focuses on these two approaches ie the parametric finite element and the fuzzy logic. In order to propose an efficient compensation of the pose errors induced by elastic deflections, the modeling and identification methods are described, tested on an industrial robot and then discussed for forming, assembly or machining applications. This allows to list the main drawbacks and advantages of each method with respect to the task and the user requirements.

The paper is organized as follows. In the first section the elasto-geometrical calibration of a robotic structure based respectively on an analytical parametric modeling and a Takagi-Sugeno fuzzy inference system is described. Next section presents their application to the KUKA IR-663 robot manipulator. Both mod-

eling methods are analyzed and discussed. Then an experimental validation of the elasto-modeling method based on the fuzzy inference system is conducted in an industrial forming context.

\section{Elasto-Geometrical calibration of a robotic structure}

In this section, the elasto-geometrical modeling of the robot's structure is done by the superposition of its geometrical and elastic models. This allows to take into account all the geometrical and elastic effects that have a significant influence onto its pose accuracy. The geometrical model is obtained in the classical way but two different approaches are developed to derive its elastic model. The first one is based on an analytical parametric modeling and the second one on a fuzzy inference system.

\subsection{Geometrical modeling}

\subsubsection{Definition of the frames}

In order to describe a robot structure in its cell, the following frames are used (Fig. 1):

- $R_{-1}$ and $R_{0}$ : the frames attached respectively to the cell and the robot's base,

- $R_{n}$ and $R_{n+1}$ : the frame attached respectively to the robot's end-effector and the tool,

- $R_{p}$ : the frame attached to the part to be manufactured.

For the modeling, the frame $R_{i}$, attached to the body $C_{i}$, is defined by:

$$
R_{i}=\left(O_{i}, \mathbf{x}_{i}, \mathbf{y}_{i}, \mathbf{z}_{i}\right) .
$$




\subsubsection{Definition of the poses controlled and reached}

In the frame $R_{-1}$, the vector that defines the tool pose ${ }^{-1} \mathbf{S}_{n+1}^{k}$ (position and orientation) that is controlled by the joint configuration $\mathbf{q}^{k}$ is defined by:

$$
{ }^{-1} \mathbf{S}_{n+1}^{k}=\left[\begin{array}{c}
{ }^{-1} \mathbf{P}_{n+1}^{k} \\
{ }^{-1} \mathbf{\Phi}_{n+1}^{k}
\end{array}\right]=f\left(\mathbf{q}^{k}, \xi\right),
$$

with:

$$
\begin{aligned}
{ }^{-1} \mathbf{P}_{n+1}^{k} & =\left[\begin{array}{lll}
{ }^{-1} P_{n+1, x}^{k} & { }^{-1} P_{n+1, y}^{k} & { }^{-1} P_{n+1, z}^{k}
\end{array}\right]^{T}, \\
{ }^{-1} \Phi_{n+1}^{k} & =\left[\begin{array}{lll}
{ }^{-1} \Phi_{n+1, x}^{k} & { }^{-1} \Phi_{n+1, y}^{k} & { }^{-1} \Phi_{n+1, z}^{k}
\end{array}\right]^{T} .
\end{aligned}
$$

The vector that defines the pose that is actually reached by the robot is:

$$
{ }^{-1} \mathbf{S}_{n+1}^{r, k}=\left[\begin{array}{c}
{ }^{-1} \mathbf{P}_{n+1}^{r, k} \\
{ }^{-1} \mathbf{\Phi}_{n+1}^{r, k}
\end{array}\right] .
$$

\subsubsection{Forward geometrical modeling}

The forward geometrical model is defined by the relation (1) that links the controlled pose ${ }^{-1} \mathbf{S}_{n+1}^{k}$ to the joint configuration $\mathbf{q}^{k}$ and the vector of the geometrical parameters $\xi$.

This model is obtained by using the product of the following homogeneous transformation matrices (Fig. 1) [10]:

$$
{ }^{-1} \mathbf{T}_{n+1}={ }^{-1} \mathbf{T}_{0}{ }^{0} \mathbf{T}_{n}{ }^{n} \mathbf{T}_{n+1}
$$

for which the homogeneous transformation ${ }^{i-1} \mathbf{T}_{i}$ giving the position, ${ }^{i-1} \mathbf{P}_{i}$, and orientation, ${ }^{i-1} \mathbf{A}_{i}$, of $R_{i}$ within $R_{i-1}$ is:

$$
{ }^{i-1} \mathbf{T}_{i}=\left[\begin{array}{ccc|c}
{ }^{i-1} \mathbf{A}_{i} & { }^{i-1} \mathbf{P}_{i} \\
\hline 0 & 0 & 0 & 1
\end{array}\right] .
$$

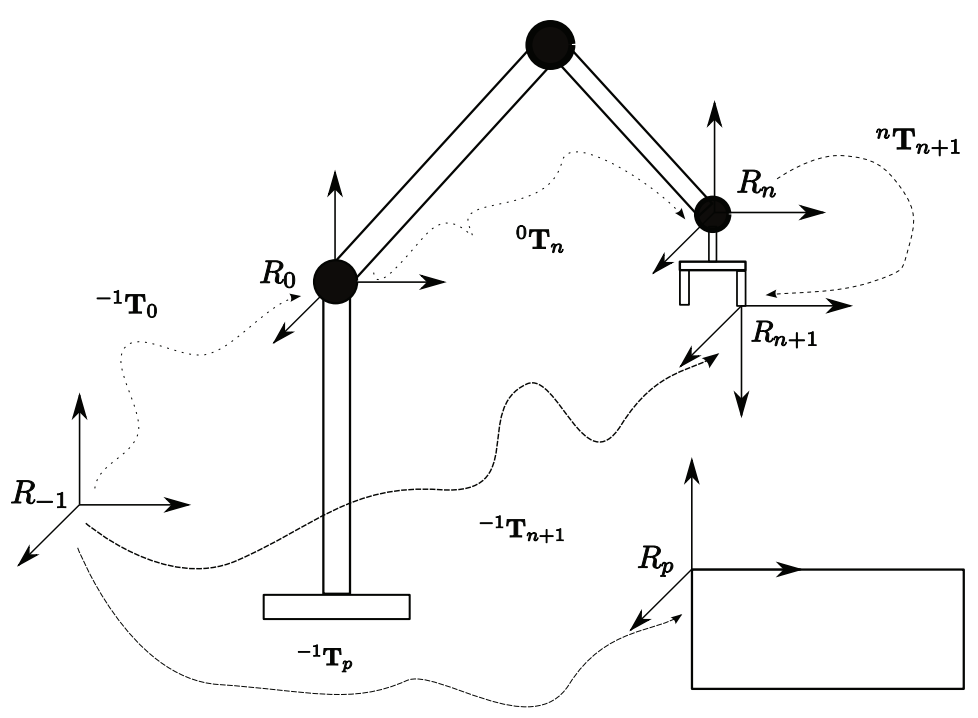

Figure 1: Frames and transformation matrices between $R_{-1}$ and $R_{n+1}$ 


\subsubsection{Inverse geometrical modeling}

The inverse geometrical model that links the joint configuration $\mathbf{q}^{k}$ to the controlled pose ${ }^{-1} \mathbf{S}_{n+1}^{k}$ and the vector of the geometrical parameters $\xi$ is noted:

$$
\mathbf{q}^{k}=g\left({ }^{-1} \mathbf{S}_{n+1}^{k}, \xi\right) \text {. }
$$

\subsubsection{Linear error model}

For the joint configuration $\mathbf{q}^{k}$, the pose error vector ${ }^{-1} d \mathbf{S}_{n+1}^{k}$, induced by the geometrical errors gathered in the vector $d \xi$ is defined by:

$$
{ }^{-1} d \mathbf{S}_{n+1}^{k}={ }^{-1} \mathbf{S}_{n+1}^{r, k}-{ }^{-1} \mathbf{S}_{n+1}^{k}=f\left(\mathbf{q}^{k}, \xi+d \xi\right)-f\left(\mathbf{q}^{k}, \xi\right) .
$$

The linear error model is obtained using the Jacobean matrix ${ }^{-1} \mathbf{J}_{n+1}^{k}$ of all geometrical parameters calculated for the robot configuration $k$ :

$$
{ }^{-1} d \mathbf{S}_{n+1}^{k}={ }^{-1} \mathbf{J}_{n+1}^{k} d \xi
$$

Using a number $n_{m}$ of robot configurations $\mathbf{q}^{k},\left(k=\left\{1, \ldots, n_{m}\right\}\right)$ and measuring the corresponding pose errors ${ }^{-1} d \mathbf{S}_{n+1}^{k}$, the global error model can be formulated as follows:

$$
\left[\begin{array}{c}
{ }^{-1} d \mathbf{S}_{n+1}^{1} \\
{ }^{-1} d \mathbf{S}_{n+1}^{2} \\
\ldots \\
{ }^{-1} d \mathbf{S}_{n+1}^{n_{m}}
\end{array}\right]=\left[\begin{array}{c}
{ }^{-1} \mathbf{J}_{n+1}^{1} \\
{ }^{-1} \mathbf{J}_{n+1}^{2} \\
\ldots \\
{ }^{-1} \mathbf{J}_{n+1}^{n_{m}}
\end{array}\right] d \xi .
$$

This leads to:

$$
{ }^{-1} d \mathbf{S}_{n+1}^{G}={ }^{-1} \mathbf{J}_{n+1}^{G} d \xi .
$$

The global Jacobian matrix ${ }^{-1} \mathbf{J}_{n+1}^{G}$ allows to study the influence of the geometrical errors onto the robot pose errors as well as their observability [41]. By removing the columns of ${ }^{-1} \mathbf{J}_{n+1}^{G}$ corresponding to the parameters that have no or a little influence onto the pose accuracy, it is possible to obtain the modified Jacobian matrix ${ }^{-1} \mathbf{J}_{n+1}^{B}$ from which the vector of identifiable errors $d \xi^{B}$ can be calculated. This can be done through the least-square method using the pseudo-inverse matrix:

$$
d \xi^{B}=\left[{ }^{-1} \mathbf{J}_{n+1}^{B}{ }^{+}\right]{ }^{-1} d \mathbf{S}_{n+1}^{B}
$$

where ${ }^{-1} \mathbf{J}_{n+1}^{B}{ }^{+}$stands for the pseudo-inverse matrix of ${ }^{-1} \mathbf{J}_{n+1}^{B}$.

The vector of identifiable parameters $d \xi^{B}$ can also be calculated in an iterative way. For that purpose, the equation (12) is formulated for the first pose measurement data only $(k=1)$ and it is solved to obtain a first least-squares estimation of the geometrical error vector $d \xi^{B}$ noted $d \xi^{B, 1}$.

$$
{ }^{-1} d \mathbf{S}_{n+1}^{B}={ }^{-1} \mathbf{J}_{n+1}^{B} d \xi^{B}
$$

The procedure is then iterated for each pose measurement until the error vector's components of $d \xi^{B, k}$ tend toward zero and the geometrical parameters of vector $\xi^{B, k}$ converge to some stable values. At each iteration $k$, the geometrical parameters are updated by adding $d \xi^{B, k}$ to the current value of $\xi^{B, k}$. The Jacobian matrix ${ }^{-1} \mathbf{J}_{n+1}^{B, k+1}$ as well as the vector $d \mathbf{S}_{n+1}^{B, k+1}$ are updated at each iteration [42]. 


\subsection{Elastic modeling}

The gravity and the applied forces elastically strain the robot's structure and the resulting elastic displacements can be calculated by the relation:

$$
{ }^{-1} d \mathbf{S}_{n+1}=\left[{ }^{-1} \mathbf{K}_{e q_{n+1}}\right]^{-1}{ }^{-1} \mathbf{F}_{e q_{n+1}}={ }^{-1} \mathbf{C}_{e q_{n+1}}{ }^{-1} \mathbf{F}_{e q_{n+1}}
$$

where:

- ${ }^{-1} \mathbf{K}_{e q_{n+1}}$ is the equivalent stiffness matrix of the robot structure calculated at the center of the tool frame $R_{n+1}$ (TCP) for the configuration $\mathbf{q}^{k}$,

- ${ }^{-1} \mathbf{F}_{e q_{n+1}}$ is the equivalent wrench acting on TCP,

- ${ }^{-1} \mathbf{C}_{e q_{n+1}}$ is the equivalent compliance matrix for the configuration $\mathbf{q}^{k}$.

Two approaches are proposed and described for the elastic modeling. They are respectively based on an analytical parametric modeling and a TakagiSugeno fuzzy inference system. For that purpose only the elastic behavior of the mechanical structure is considered. Measurements of the TCP's elastic displacements have been conducted with the controller on and off (actuators blocked) and have shown exactly the same elastic behavior of the robot. As a results, we can assume that the robot controller does not compensate the elastic displacements and do not have to be integrated in the elastic models.

\subsubsection{Analytical parametric modeling}

Description of the structure. In order to derive the elastic model, the robot is first described as a set of nodes corresponding to the characteristic points of its structure (ends of links, centers of frames used to perform the geometrical modeling, joint centers). These nodes are set to define the elastic beams used to describe bodies, joints and connections with the environment (Fig. 3).

Nodal wrenches and nodal displacements. The vector of nodal wrench, $\mathbf{F}_{v}$, applied at the node $v$ is:

$$
\mathbf{F}_{v}=\left[\begin{array}{c}
\mathbf{f}_{v} \\
\mathbf{m}_{v}
\end{array}\right]=\left[\begin{array}{llllll}
f_{v, x} & f_{v, z} & f_{v, z} & m_{v, x} & m_{v, z} & m_{v, z}
\end{array}\right]^{T} .
$$

Expressed in $R_{u, v}$ and $R_{-1}$, the wrench $\mathbf{F}_{v}$ will respectively be written ${ }^{u, v} \mathbf{F}_{v}$ and ${ }^{-1} \mathbf{F}_{v} . R_{u, v}$ is the local frame attached to the beam defined between $u$ and $v$ with $\mathbf{x}_{u, v}$ along the neutral fiber $(u, v)$ (Fig. 2). The nodal displacements resulting of the application of $\mathbf{F}_{v}$ at the node $v$ are:

$$
d \mathbf{S}_{v}=\left[\begin{array}{l}
d \mathbf{P}_{v} \\
d \mathbf{\Phi}_{v}
\end{array}\right]
$$

with:

- $d \mathbf{P}_{v}=\left[\begin{array}{lll}d P_{v, x} & d P_{v, y} & d P_{v, z}\end{array}\right]^{T}$, the translational displacement vector of $v$ and,

- $d \boldsymbol{\Phi}_{v}=\left[\begin{array}{lll}d \phi_{v, x} & d \phi_{v, y} & d \phi_{v, z}\end{array}\right]^{T}$, the vector gathering the three rotation angles of the beam's section. 


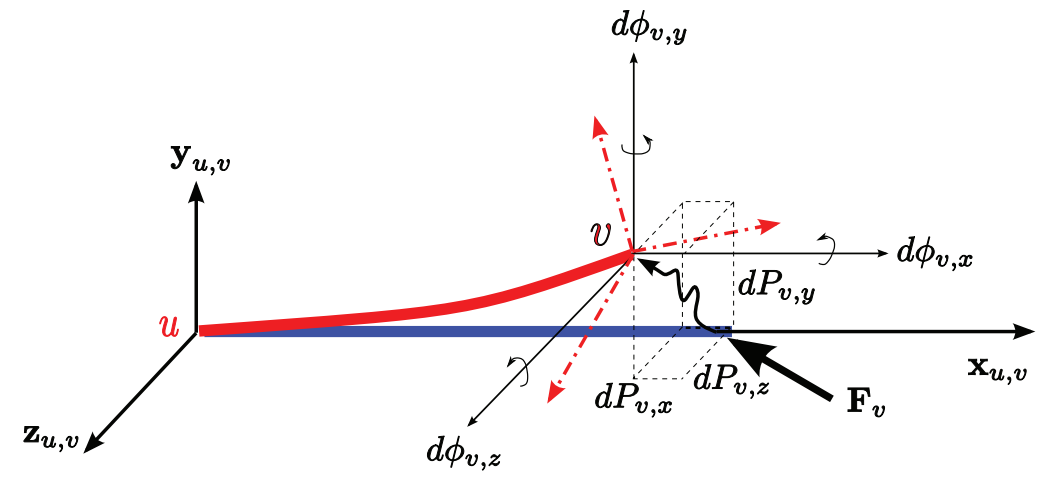

Figure 2: Elastic beam, nodal wrench and displacement

Stiffness matrices. The stiffness matrix associated to the beam defined between the nodes $u$ and $v$ is:

$$
{ }^{u, v} \mathbf{K}_{u, v}=\left[\begin{array}{c|c}
{ }^{u, v} \mathbf{K}_{u, v}^{11} & { }^{u, v} \mathbf{K}_{u, v}^{12} \\
\hline{ }^{u, v} \mathbf{K}_{u, v}^{21} & { }^{u, v} \mathbf{K}_{u, v}^{22}
\end{array}\right]_{(12 \times 12)} .
$$

The sub-matrices ${ }^{u, v} \mathbf{K}_{u, v}^{11},{ }^{u, v} \mathbf{K}_{u, v}^{12},{ }^{u, v} \mathbf{K}_{u, v}^{21}$ and ${ }^{u, v} \mathbf{K}_{u, v}^{22}$ are defined in [32]. All mechanical parameters involved in the calculation of these matrices describing the robot structure are gathered in the vector $\boldsymbol{\Lambda}$ (the length and the section of the beams, the Young and the Coulomb modulus, the quadratic and the polar momentum).

Modeling of joint stiffness. The stiffness of the robot's joints are also described using elastic elements with their associated stiffness matrices. For example, the stiffness of the compliant joint defined between the nodes $k$ and $l$ and which axis is along the $\mathbf{x}_{k, l}$ of the frame $R_{k, l}$ is described by the $(12 \times 12)$ matrix:

$$
{ }^{k, l} \mathbf{K}_{k, l}=\left[\begin{array}{c|c}
{ }^{k, l} \mathbf{K}_{k, l}^{D} & -{ }^{k, l} \mathbf{K}_{k, l}^{D} \\
\hline-^{k, l} \mathbf{K}_{k, l}^{D} & { }^{k, l} \mathbf{K}_{k, l}^{D}
\end{array}\right]_{(12 \times 12)}
$$

where:

$$
{ }^{k, l} \mathbf{K}_{k, l}^{D}=\operatorname{diag}\left[\begin{array}{llllll}
K_{a} & K_{r} & K_{r} & K_{a r} & K_{r r} & K_{r r}
\end{array}\right]_{(6 \times 6)} .
$$

$K_{a}$ and $K_{r}$ respectively stand for the axial and radial translational stiffnesses. $K_{a r}$ and $K_{r r}$ are the axial and radial rotational stiffnesses [43]. All the joint's stiffness parameters of the robot's structure will further be gathered in the vector $\boldsymbol{\Gamma}$.

Assembly of the global stiffness matrix. In order to calculate the global stiffness of the robot's structure, the stiffness matrices of all links and joints have to be expressed in the same frame. The calculation in $R_{-1}$ of the stiffness matrix components of the beam $(u, v)$ is done using the transformation matrix $\mathbf{B}_{u, v}$ according to the relation:

$$
{ }^{-1} \mathbf{K}_{u, v}=\left[\mathbf{B}_{u, v}\right]^{-1-1} \mathbf{K}_{u, v} \mathbf{B}_{u, v}=\left[\begin{array}{c|c}
{ }^{-1} \mathbf{K}_{u, v}^{11} & { }^{-1} \mathbf{K}_{u, v}^{12} \\
\hline{ }^{u, v} \mathbf{K}_{u, v}^{21} & { }^{-1} \mathbf{K}_{u, v}^{22}
\end{array}\right]
$$


for which:

$$
\mathbf{B}_{u, v}=\left[\begin{array}{cccc}
\mathbf{A}_{u, v} & \mathbf{0}_{3,3} & \mathbf{0}_{3,3} & \mathbf{0}_{3,3} \\
\mathbf{0}_{3,3} & \mathbf{A}_{u, v} & \mathbf{0}_{3,3} & \mathbf{0}_{3,3} \\
\mathbf{0}_{3,3} & \mathbf{0}_{3,3} & \mathbf{A}_{u, v} & \mathbf{0}_{3,3} \\
\mathbf{0}_{3,3} & \mathbf{0}_{3,3} & \mathbf{0}_{3,3} & \mathbf{A}_{u, v}
\end{array}\right]_{(12 \times 12)}
$$

$\mathbf{A}_{u, v}$ is the cosine matrix used to describe the orientation of the frame $R_{u, v}$ with respect to $R_{-1}$. The assembly of all matrices is then performed by adding the effects at all nodes according to their labels [32] (the ${ }^{-1}$ indices of each sub-matrix have been omitted for clarity). For the structure described by Fig. 3 this leads to the following global stiffness matrix ${ }^{-1} \mathbf{K}_{G}$ (21).

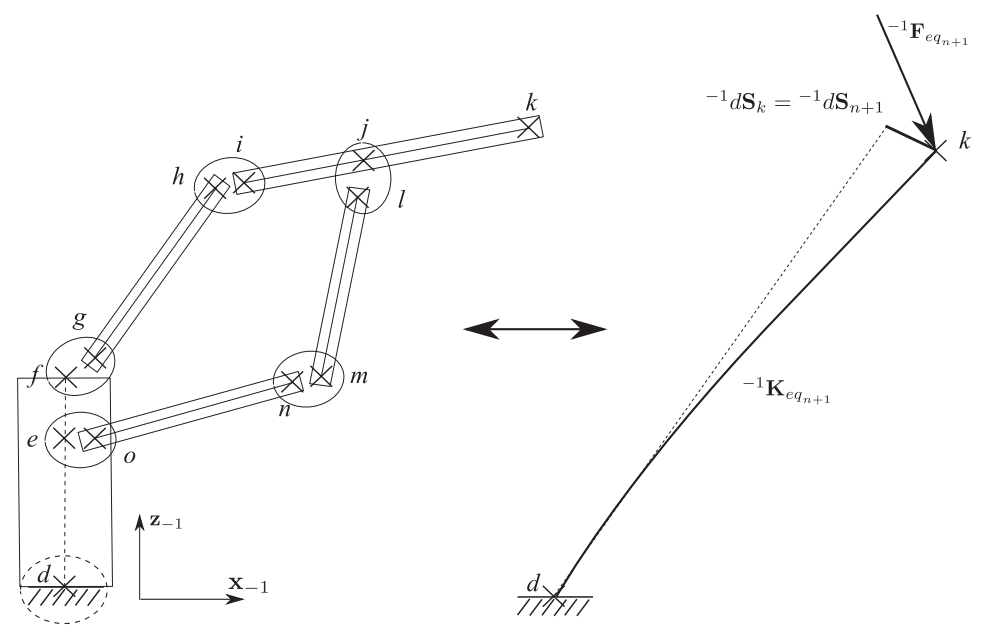

Figure 3: Elastic modeling and equivalent (beam) structure

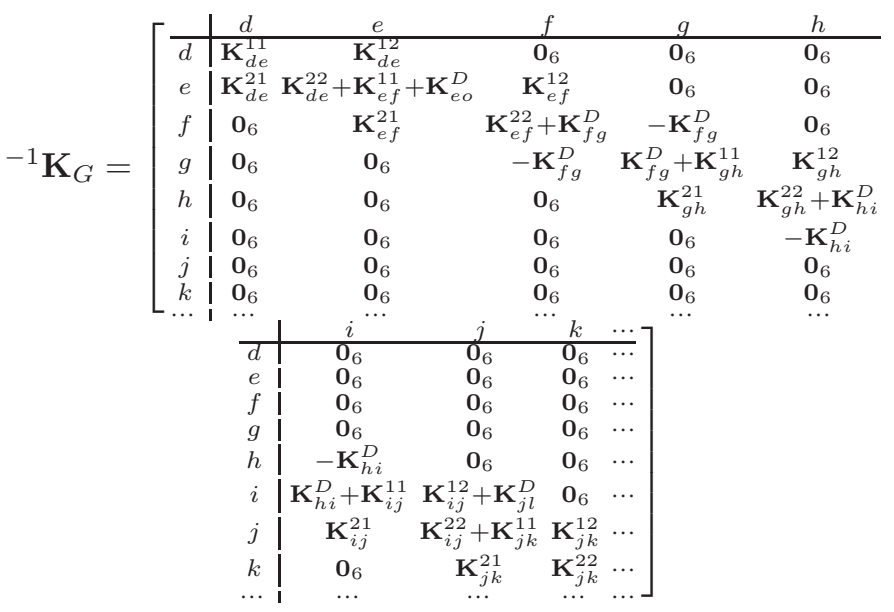

We defined:

- ${ }^{-1} \mathbf{F}_{G}=\left[\begin{array}{lll}{ }^{-1} \mathbf{F}_{d}^{T} & \cdots & { }^{-1} \mathbf{F}_{o}^{T}\end{array}\right]^{T}$, the vector obtained by the concatenation of all wrenches that act on the structure's nodes,

- ${ }^{-1} d \mathbf{S}_{G}=\left[\begin{array}{lll}{ }^{-1} d \mathbf{S}_{d}^{T} & \cdots & { }^{-1} d \mathbf{S}_{o}^{T}\end{array}\right]^{T}$, the corresponding vector of the nodal displacements. 
The forces due to the own weight of all bodies also induce elastic deformations of the robot's structure. Therefore they have to be merged in the vector ${ }^{-1} \mathbf{F}_{G}$. For that purpose, equivalent forces and torques due to gravity are calculated for each body at their respective nodes [44]. Moreover, the conditions imposed by the links between the structure and the environment (ground, machine frame, etc.) have to be considered. These boundary conditions correspond to the nodes for which some translational and/or rotational displacements are nil. This is expressed in the global stiffness matrix ${ }^{-1} \mathbf{K}_{G}$ as well as in the vectors ${ }^{-1} \mathbf{F}_{G}$ and ${ }^{-1} d \mathbf{S}_{G}$ by removing the columns and lines corresponding to the nodes with nil displacements. This leads to the stiffness matrix ${ }^{-1} \mathbf{K}_{C}$ and to the vectors ${ }^{-1} \mathbf{F}_{C}$ et ${ }^{-1} d \mathbf{S}_{C}$.

For the example of Fig. 3, the boundary conditions at node $d$ are due to the embedment of the robot into the ground. Therefore, the resulting translational and rotational displacements of the node $d$ are zero. This gives for ${ }^{-1} \mathbf{K}_{C}$ :

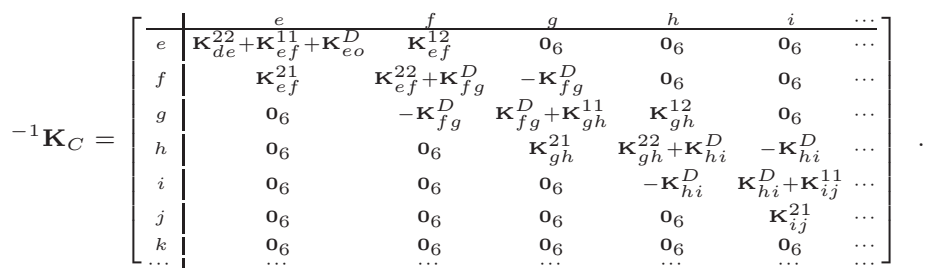

The vectors ${ }^{-1} \mathbf{F}_{C}$ and ${ }^{-1} d \mathbf{S}_{C}$ are:

$$
\begin{aligned}
& -{ }^{-1} \mathbf{F}_{C}=\left[\begin{array}{lll}
{ }^{-1} \mathbf{F}_{e}^{T} & \ldots & { }^{-1} \mathbf{F}_{o}^{T}
\end{array}\right]^{T}, \\
& \text { - }{ }^{-1} d \mathbf{S}_{C}=\left[\begin{array}{llll}
{ }^{-1} d \mathbf{S}_{e}^{T} & \cdots & { }^{-1} d \mathbf{S}_{o}^{T}
\end{array}\right]^{T} .
\end{aligned}
$$

Since the matrix ${ }^{-1} \mathbf{K}_{C}$ is definite positive, its inversion allows the calculation of the nodal displacements vector ${ }^{-1} d \mathbf{S}_{C}$ due to the wrench ${ }^{-1} \mathbf{F}_{C}$ applied on the structure. The elastic parametric model taking into account boundary conditions then becomes:

$$
{ }^{-1} \mathbf{F}_{C}={ }^{-1} \mathbf{K}_{C} \cdot{ }^{-1} d \mathbf{S}_{C}
$$

Sub-structure and equivalent stiffness model. The elastic displacements can be calculated for a given load of the structure (body own weight, payload, external forces, etc.). It is possible to obtain them at the node corresponding to the end-effector frame center, ${ }^{-1} d \mathbf{S}_{n}$, or at the TCP, ${ }^{-1} d \mathbf{S}_{n+1}$. The whole elastic behavior of the structure is described by an equivalent elastic beam of stiffness matrix ${ }^{-1} \mathbf{K}_{e q_{n+1}}$ and an equivalent wrench ${ }^{-1} \mathbf{F}_{e q_{n+1}}$ of the loading case. For the previous structure (Fig. 3), this leads to find the equivalent beam between the nodes $d$ et $k$. In the general case, the stiffness matrix and the vector of nodal wrenches and displacements are defined between the base and the TCP nodes using the following partition matrices:

$$
\left[\begin{array}{c|c}
{ }^{-1} \mathbf{K}_{R, R} & { }^{-1} \mathbf{K}_{R, n+1} \\
\hline{ }^{-1} \mathbf{K}_{n+1, R} & { }^{-1} \mathbf{K}_{n+1, n+1}
\end{array}\right]\left[\begin{array}{c}
{ }^{-1} d \mathbf{S}_{R} \\
\hline{ }^{-1} d \mathbf{S}_{n+1}
\end{array}\right]=\left[\frac{{ }^{-1} \mathbf{F}_{R}}{{ }^{-1} \mathbf{F}_{n+1}}\right] .
$$

The index $R$ corresponds to the components of all nodes excepted the one of the TCP, $n+1$. Expanding the previous relations, one can obtain: 


$$
\left\{\begin{array}{l}
{ }^{-1} \mathbf{K}_{R, R}{ }^{-1} d \mathbf{S}_{R}+{ }^{-1} \mathbf{K}_{R, n+1}{ }^{-1} d \mathbf{S}_{n+1}={ }^{-1} \mathbf{F}_{R} \\
{ }^{-1} \mathbf{K}_{n+1, R}{ }^{-1} d \mathbf{S}_{R}+{ }^{-1} \mathbf{K}_{n+1, n+1}{ }^{-1} d \mathbf{S}_{n+1}={ }^{-1} \mathbf{F}_{n+1}
\end{array}\right.
$$

The vector of nodal displacements ${ }^{-1} d \mathbf{S}_{n+1}$ at the TCP can then be calculated according to:

$$
{ }^{-1} d \mathbf{S}_{n+1}=\left[{ }^{-1} \mathbf{K}_{e q_{n+1}}\right]^{-1}{ }^{-1} \mathbf{F}_{e q_{n+1}}
$$

with:

$$
\begin{aligned}
& { }^{-1} \mathbf{F}_{e q_{n+1}}={ }^{-1} \mathbf{F}_{n+1}-{ }^{-1} \mathbf{K}_{n+1, R}{ }^{-1}\left(\mathbf{K}_{R, R}\right)^{-1-1} \mathbf{F}_{R}, \\
& { }^{-1} \mathbf{K}_{e q_{n+1}}={ }^{-1} \mathbf{K}_{n+1, n+1}-{ }^{-1} \mathbf{K}_{n+1, R}\left({ }^{-1} \mathbf{K}_{R, R}\right)^{-1}-\mathbf{K}_{R, n+1} .
\end{aligned}
$$

The equation (26) defines the equivalent parametric Direct Elastic Model of the structure which can be described by the general relation:

$$
{ }^{-1} d \mathbf{S}_{n+1}=h\left(\mathbf{q}^{k}, \xi,{ }^{-1} \mathbf{F}_{e q_{n+1}}, \boldsymbol{\Lambda}, \boldsymbol{\Gamma}\right) .
$$

Elastic parameters identification. This consists in the estimation of the structure's stiffness parameters independently of the geometrical parameter errors because their effects on the elastic behavior are negligible. We define ${ }^{-1} d \mathbf{S}_{n+1}^{m, k}$ and ${ }^{-1} d \mathbf{S}_{n+1}^{k}$ the vectors of the displacements measured and calculated, therefore, for a pose and a wrench $k$, the position and orientation errors ${ }^{-1} E_{P, n+1}^{k}$ and ${ }^{-1} E_{\phi, n+1}^{k}$ are respectively:

$$
\begin{aligned}
& { }^{-1} E_{P, n+1}^{k}=\left\|{ }^{-1} \mathbf{P}_{n+1}^{m, k}-{ }^{-1} \mathbf{P}_{n+1}^{k}\right\|, \\
& { }^{-1} E_{\phi, n+1}^{k}=\left\|{ }^{-1} \boldsymbol{\Phi}_{n+1}^{m, k}-{ }^{-1} \boldsymbol{\Phi}_{n+1}^{k}\right\| .
\end{aligned}
$$

Considering $n_{m}$ measurement points, the error functions used to identify the parameters of the elastic model are:

$$
\begin{aligned}
& { }^{-1} E_{P, n+1}=\frac{1}{n_{m}} \sum_{k=1}^{n_{m}}\left({ }^{-1} E_{P, n+1}^{k}\right), \\
& { }^{-1} E_{\phi, n+1}=\frac{1}{n_{m}} \sum_{k=1}^{n_{m}}\left({ }^{-1} E_{\phi, n+1}^{k}\right) .
\end{aligned}
$$

The identification of the elastic parameters can be done using either one of the two error functions or by using both through a multi-objective optimization scheme. Another way is to normalize position and orientation contributions (units or weight factors) in a single criterion. Moreover, to insure the creation of a valid model, the measurement data used to identify these parameters must thoroughly cover the operating range corresponding to the process (machining, forming, etc.). 


\subsubsection{Fuzzy logic modeling}

This section presents the application of fuzzy logic modeling to the elastic behavior identification from measured data. Fuzzy set theory is used in datadriven extraction of rule-based models. This concept is based on the fact that Tagagi-Sugeno fuzzy model provides an universal approximation property [45, 36, 46, 38, 47, 39]. Fuzzy Logic (FL) was developed by Zadeh [48]. Basically, FL is a multivaluated logic that allows intermediate values to be defined between conventional boolean values. Notions like "rather tall" or "very fast" may be formulated mathematically and processed by computers in order to apply a more human-like way of thinking in programming. Fuzzy inference is the actual mapping process from a given set of input/outputs measurements, based on a set of fuzzy rules.

The input variables are fuzzified by considering convenient linguistic subsets such as "medium", "low" or "heavy". In order to guarantee the completeness and the semantics of the rules, strong triangular fuzzy partitions are used (Fig. 4). A strong fuzzy partition of an input $x_{j}$ is defined by:

$$
\forall x_{j} \quad \sum_{j} \mu_{A_{j}^{k_{j}}}\left(x_{j}\right)=1
$$

where: $x_{j}, j=\{1, . ., n\}$ are the $n$ inputs of the model; $A_{j}^{k_{j}}, k_{j}=\left\{1, \ldots, m_{j}\right\}$ are the $m_{j}$ fuzzy sets and $\mu_{A_{j}^{k_{j}}}\left(x_{j}\right)$ is the membership degree of input $x_{j}$ to the fuzzy subset $A_{j}^{k_{j}}$.

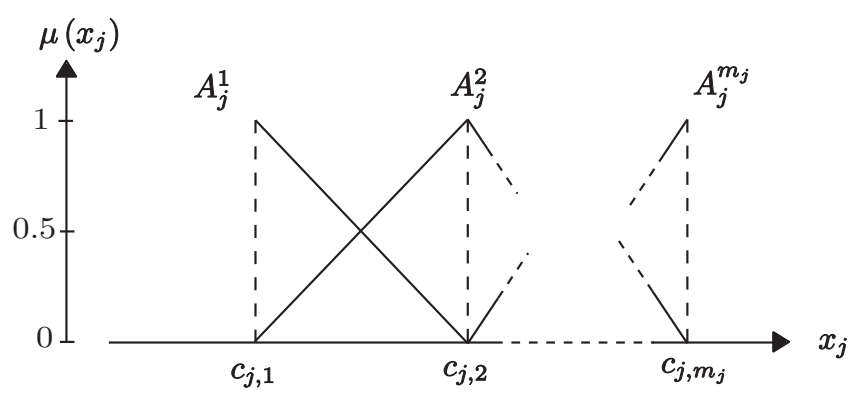

Figure 4: Strong fuzzy partition with triangular Membership Functions

Triangular form is chosen for the strong fuzzy partition since the number of parameters necessary to describe the partitioning of an input is the lowest possible. Thus the identification of the FL model will be easier. With the notations of Fig. 4, the partition is totaly defined by the modal values of the triangles $\left\{c_{j, 1}, \ldots, c_{j, m_{j}}\right\}$. As the Membership Functions (MFs) cross at $\mu\left(x_{j}\right)=0.5$, the completeness is guaranteed. The semantics is guaranteed under the condition:

$$
c_{j, 1}<c_{j, 2}<\ldots<c_{j, m_{j}} .
$$

Transition between fuzzy input sets and output is determined by a group of fuzzy If-Then rules constructed on an expert knowledge and/or on the available data. The general form of the $i^{\text {th }}$ rule of a system containing $N$ rules is:

$$
\text { If } x_{1} \text { is } \mathrm{A}_{1}^{\mathrm{k}_{1}} \text { and } \ldots \text { and } \mathrm{x}_{\mathrm{n}} \text { is } \mathrm{A}_{\mathrm{n}}^{\mathrm{k}_{\mathrm{n}}} \text { then } \mathrm{y}=\mathrm{b}^{\mathrm{i}}
$$


where $\mathrm{b}^{i}$ is a real value defining the conclusion of the rule. The fuzzy input MFs are combined with a logical and. The name given to these combinations is the premises of the rules. The output of a FL model is calculated using the membership degrees of each input to the MFs. For an input vector $\mathbf{x}$ the output $y$ is obtained as a weighted average of the rules' contribution:

$$
y(\mathbf{x})=\frac{\sum_{i=1}^{N} \alpha_{i}(\mathbf{x}) \times \mathrm{b}^{i}}{\sum_{j=1}^{N} \alpha_{j}(\mathbf{x})}
$$

where $\alpha_{i}$ is the firing strength of the rule $i$ for the observation $\mathbf{x}$. Since the product is used as conjunction operator, $\alpha_{i}$ value is computed by:

$$
\alpha_{i}(\mathbf{x})=\prod_{j=1}^{n} \mu_{A_{j}^{k_{j}}}\left(x_{j}\right) .
$$

A multi-output fuzzy inference system is developed to describe the elastic behavior of a robot in position as well as in orientation over the global workspace. The driven joint coordinates of vector $\mathbf{q}^{k}$ and the external wrench acting on the TCP ${ }^{-1} \mathbf{F}_{e q_{n+1}}$ are the inputs. The outputs are the six components of the TCP elastic displacement vector ${ }^{-1} d \mathbf{S}_{n+1}$ computed using the equivalent compliance matrix, ${ }^{-1} \mathbf{C}_{e q_{n+1}}$ :

$$
{ }^{-1} d \mathbf{S}_{n+1}={ }^{-1} \mathbf{C}_{e q_{n+1}}{ }^{-1} \mathbf{F}_{e q_{n+1}}=l\left(\mathbf{q}_{k}, \mathbf{c}_{\{1 \ldots n\},\left\{m_{1} \ldots m_{n}\right\}}, \mathcal{A}, \mathcal{T}\right) .
$$

Since the Takagi-Sugeno model of order 0 allows a single output, the problem space for the identification of the compliance matrix is partitioned into smaller and easily tractable subspaces. Each component of the matrix is considered as a single fuzzy output system.

An algorithm for data-driven identification, structure optimization and rule extraction of the FL model is introduced. The FL model is entirely identified from the measured displacements obtained for different poses over the workspace and in the case of external wrenches corresponding to the application. Two different databases are used in the identification process, namely the training set $\mathcal{A}$ and the test set $\mathcal{T}$. The test set is used to prevent over-learning of the FL model which would imply a specialization in a particular area of the input space.

With the training and test data established, a quasi-automated identification program processes the data to create each individual FL model in order to predict the compliance behavior of the manipulator. Before training occurs, an initial FL model is created with two MFs per input $\left(m_{j}=2\right)$. Then, the problem of FL model identification may be divided into three iterative steps:

i) In a first approximation the input domain is automatically divided into $\left(m_{j}-1\right)$ equal parts and triangular strong fuzzy partitions are generated (Fig. 4). This initialization of MFs placement $\left(c_{j, 1}, \ldots, c_{j, m_{j}}, j=\{1, . ., n\}\right)$ gives an initial solution. The initialization of the conclusions of the rules is achieved using a Rapid Prototyping Algorithm (RPA) [49, 50]. This method is based on the properties of the grid defined by the partitioning of the input space. This algorithm allows an automatic extraction of a first set of rules. A sub-optimal solution for the placement of MFs is found by using a modified version of the method proposed by Solis and Wets [51]. 
ii) For this structure and MFs placement, a Gradient Descent method is used for the optimization of the vector of fuzzy rule conclusions $\mathbf{b}$ by minimizing the quadratic difference between the measured and calculated TCP poses.

iii) A systematic method is used to determine an enhanced structure for the FL models. The simplest possible structure is initialized (i.e. $m_{j}=2$ for each input). The complexity is then increased in a systematic way: MFs are added to the inputs while a suitable compromise between the performance and the size of the fuzzy rule basis is obtained [52].

The major difficulty is to derive a fuzzy model with a good trade-off between the approximation quality, the complexity and the clarity of the model [40].

\subsection{Elasto-geometrical calibration of a structure}

Based on the geometrical and elastic models obtained, the elasto-geometrical model of the robot's structure can be calculated either by relation (39) using the analytical parametric approach or by relation (40) using the fuzzy description.

$$
\begin{aligned}
{ }^{-1} \mathbf{S}_{n+1} & =f\left(\mathbf{q}^{k}, \xi\right)+h\left(\mathbf{q}^{k}, \xi,{ }^{-1} \mathbf{F}_{e q_{n+1}}, \boldsymbol{\Lambda}, \boldsymbol{\Gamma}\right) \\
& =f\left(\mathbf{q}^{k}, \xi\right)+l\left(\mathbf{q}^{k}, \mathbf{c}_{\{1 . . n\},\left\{m_{1} \ldots m_{n}\right\}}, \mathcal{A}, \mathcal{T}\right)
\end{aligned}
$$

\section{Elasto-Geometrical calibration of the KUKA-IR663}

The KUKA-IR663 hybrid robot on which the study focuses is first presented. Then the systematic approaches that have been proposed before are developed to derive the elasto-geometrical models of its structure. Simulations and experimental tests are presented in order to evaluate the limitations and the benefits of the proposed methods.

\subsection{System description and measurement method}

The KUKA-IR663 is shown on Fig. 5. This robot has been chosen for the study because:

- The closed-loop kinematic chain of its structure increases the global stiffness of the robot which fits well with the applications that we are interested in.

- To our thinking it is one of the most complicated structures of industrial robots that can be modeled [4].

Its kinematic structure can be described by Fig. 5 and 6 . This robot has a special wrist made of 4 revolute joints. Two of them are coupled (biconic wrist). The wrist is carried by an articulated mechanical structure with 6 revolute joints. If $n$ denotes the number of links excluding the one attached to the ground, the global robot structure has thus a number $l=10$ joints and $n+1=10$ links where link 0 is the fixed base and $B=l-n=1$ closed loop. This loop is a fourbar linkage; two of those links are actuated by slider-crank type mechanisms. The number $N$ of active joints is equal to 6 which corresponds to the robot's degrees of freedom number.

The experimental setup consists of a flexible multi-camera system for a precise tracking of the moving end-effector. A loading device is connected to the robot and involved to exert forces along the different axis of the manipulator 


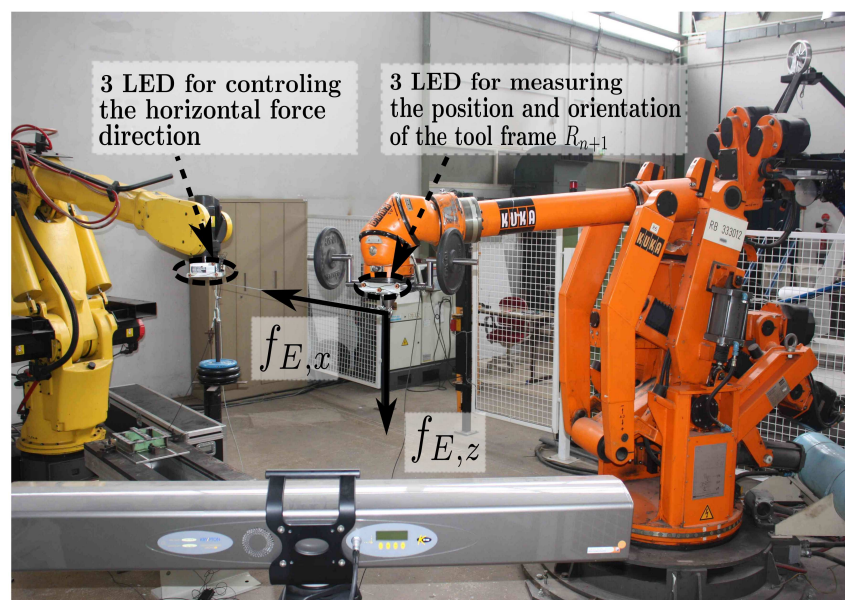

Figure 5: KUKA-IR663 Industrial robot and the experimental setup for elastic calibration

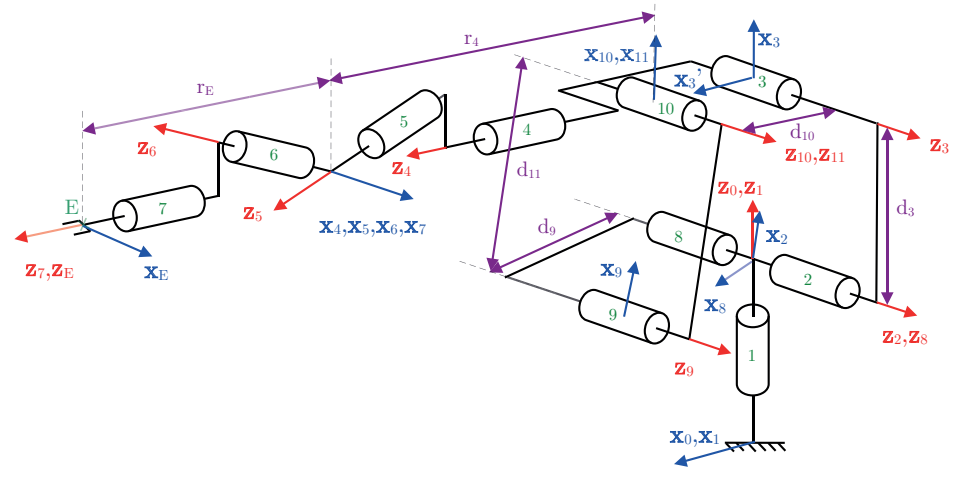

Figure 6: Kinematics of KUKA-IR663

base frame (Fig. 5). Horizontal forces are generated by deadweight suspended to the free-end of a cable guided by a pulley mounted on the end-effector of a FANUC S420iF available in the cell. Deadweight is suspended directly to the end-effector of the KUKA-IR663 in order to create vertical payload. The measurement device is the photogrammetric Nikon Metrology K600-10 system ${ }^{1}$ based on three CCD linear cameras and infra-red light active LED. Three or more LED are attached to the object and their positions are measured simultaneously by the camera. This allows the simultaneous multiple point tracking and the measurement of both, position and orientation. The system has a position measuring accuracy up to $37 \mu \mathrm{m}$ for a single point. In this work, the system is first involved to identify the transformation linking the measuring system frame to the robot base frame. Therefore, the robot TCP pose measurements, required for the model identification, are all expressed in the robot base frame $R_{0}$.

\footnotetext{
${ }^{1}$ http://www.nikonmetrology.com/optical__cmm/
} 


\subsection{Geometrical calibration of the KUKA-IR663}

\subsubsection{Derivation of the Forward and Inverse Geometrical Models}

In order to achieve a systematic procedure, Khalil and Kleinfinger's method [10] is used to establish the parametric elasto-geometrical model. This method is derived from the well-known Denavit and Hartenberg's notation and can be used to describe, with a minimum set of parameters, the open- and closed-loop robots. This notation is based on a specific frame definition, detailed in [10] and [53]. On each body $C_{i}$, a frame $R_{i}$ is defined for which, $\mathbf{z}_{i}$ axis is aligned with the axis of joint $\mathrm{i}$ and $\mathbf{x}_{i}$ is along the common normal to $\mathbf{z}_{i}$ and $\mathbf{z}_{i+1}$. Therefore, the transformation from $R_{i}$ with respect to the previous frame $R_{a(i)}$ is defined by the homogeneous matrices ${ }^{a(i)} \mathbf{T}_{i}$ :

$$
\begin{array}{r}
{ }^{a(i)} \mathbf{T}_{i}=\operatorname{rot}\left(\mathbf{z}, \gamma_{i}\right) \operatorname{trans}\left(\mathbf{z}, b_{i}\right) \operatorname{rot}\left(\mathbf{x}, \alpha_{i}\right) \\
\operatorname{trans}\left(\mathbf{x}, d_{i}\right) \operatorname{rot}\left(\mathbf{z}, \theta_{i}\right) \operatorname{trans}\left(\mathbf{z}, r_{i}\right)
\end{array}
$$

where $\gamma_{i}, \alpha_{i}$ and $\theta_{i}$ are angles and $b_{i}, d_{i}$ and $r_{i}$ are distances. Three more parameters are added for closed-loop structures. The binary variable $\sigma_{i}$, is equal to one if the joint $i$ is a prismatic and zero if it is a rotoid. The binary variable $\mu_{i}$, is equal to one if the joint $i$ is motorized and zero otherwise. The variable $a(i)$ specifies the label of the body preceding the body $C_{i}$. The geometry of the manipulator's kinematic chain can thus be defined by Table 1 .

\begin{tabular}{|r|r|r|r|r|r|r|r|r|r|}
\hline $\mathrm{i}$ & $a(i)$ & $\mu_{i}$ & $\sigma_{i}$ & $\gamma_{i}$ & $b_{i}$ & $\alpha_{i}$ & $d_{i}$ & $\theta_{i}$ & $r_{i}$ \\
\hline \hline 1 & 0 & 1 & 0 & 0 & 0 & 0 & 0 & $\theta_{1}$ & 0 \\
\hline 2 & 1 & 1 & 0 & 0 & 0 & $-\frac{\pi}{2}$ & 0 & $\theta_{2}-\frac{\pi}{2}$ & 0 \\
\hline 3 & 2 & 0 & 0 & 0 & 0 & 0 & $d_{3}$ & $\theta_{3}$ & 0 \\
\hline 4 & 3 & 1 & 0 & 0 & 0 & $-\frac{\pi}{2}$ & 0 & $\theta_{4}-\frac{\pi}{2}$ & $r_{4}+d_{10}$ \\
\hline 5 & 4 & 1 & 0 & 0 & 0 & $\frac{\pi}{6}$ & 0 & $\theta_{5}$ & 0 \\
\hline 6 & 5 & 0 & 0 & 0 & 0 & $-\frac{\pi}{3}$ & 0 & $\theta_{6}=-\theta_{5}$ & 0 \\
\hline 7 & 6 & 1 & 0 & 0 & 0 & $\frac{\pi}{6}$ & 0 & $\theta_{7}$ & 0 \\
\hline 8 & 1 & 1 & 0 & 0 & 0 & $-\frac{\pi}{2}$ & 0 & $\theta_{8}$ & 0 \\
\hline 9 & 8 & 0 & 0 & 0 & 0 & 0 & $d_{9}$ & $\theta_{9}$ & 0 \\
\hline 10 & 3 & 0 & 0 & $\frac{\pi}{2}$ & 0 & 0 & $d_{10}$ & $\theta_{10}-\frac{\pi}{2}$ & 0 \\
\hline 11 & 0 & 0 & 0 & 0 & 0 & 0 & $d_{11}$ & $\theta_{11}=0$ & 0 \\
\hline $\mathrm{E}$ & 0 & 0 & 0 & 0 & 0 & 0 & 0 & 0 & $r_{E}$ \\
\hline
\end{tabular}

Table 1: Geometrical Parameters of the KUKA-IR663

The global structure is first described by an equivalent tree structure. This structure is obtained by cutting the closed loop at joint 10 (Fig. 6). The total number of frames is equal to $n+1+2 B=12\left(R_{0}=R_{-1}\right.$ is the reference frame) since 2 frames have been added while cutting the joint 10 . It is to be noted that the geometric parameters used to locate the frame 11 relatively to the frame 9 are constants. The pose of the TCP frame $R_{n+1}$ is referred as $R_{E}$ in the following. The nominal values for the geometrical parameters of the KUKA-IR663 are given in Table 2.

\begin{tabular}{|c|c|c|c|c|c|c|c|}
\hline Parameter & $r_{1}$ & $d_{3}$ & $r_{4}$ & $d_{9}$ & $d_{10}$ & $d_{11}$ & $r_{E}$ \\
\hline \hline Nominal Value $(\mathrm{mm})$ & 885 & 1047 & 1760 & 473 & 400 & 1212 & 244 \\
\hline
\end{tabular}

Table 2: Values of Geometrical Parameters of the KUKA-IR663

In order to calculate the Forward and Inverse Geometrical Models of the robot (respectively functions $f(1)$ and $g(6)$ ), one has to establish the mathematical relation connecting the pose ${ }^{0} \mathbf{S}_{E}^{k}$ of the frame $R_{E}$ attached to the TCP 
to the configuration of the actuated joints $\mathbf{q}^{k}(47)$. For that purpose, one open kinematic chain has been selected after cutting the joint 10 .

The relation from which the functions $f$ and $g$ are deduced is obtained by multiplying the homogeneous matrices describing the relative pose of each robot's frames:

$$
{ }^{0} \mathbf{T}_{E}^{k}={ }^{0} \mathbf{T}_{1}\left(\theta_{1}^{k}\right)^{1} \mathbf{T}_{2}\left(\theta_{2}^{k}\right)^{2} \mathbf{T}_{3}\left(\theta_{3}^{k}\right)^{3} \mathbf{T}_{4}\left(\theta_{4}^{k}\right)^{4} \mathbf{T}_{5}\left(\theta_{5}^{k}\right)^{5} \mathbf{T}_{6}\left(\theta_{6}^{k}\right)^{6} \mathbf{T}_{7}\left(\theta_{7}^{k}\right)^{7} \mathbf{T}_{E} .
$$

The matrix ${ }^{0} \mathbf{T}_{E}^{k}$ gives the pose of the frame $R_{E}$ within $R_{0}$. However, as one can see (Table 1), the joints 3 and 6 are passive and relations linking their values to the actuated joints values of the robot have to be calculated. The relation (43) links the rotation angles of the wrist joints 5 and 6 .

$$
\theta_{6}^{k}=-\theta_{5}^{k}
$$

Then relation (44) is derived in order to link the value of $\theta_{3}^{k}$ to the actuated joint values $\theta_{2}^{k}, \theta_{8}^{k}$ and the geometrical parameters of the four-bar linkage $d_{3}$, $d_{9}, d_{10}$ and $d_{11}$.

$$
\theta_{3}^{k}=U\left(\theta_{2}^{k}, \theta_{8}^{k}, d_{3}, d_{9}, d_{10}, d_{11}\right)
$$

Moreover, as the joints 2 and 8 are actuated by slider-crank mechanisms, the relations (45) and (46) link the actuated translational displacements $r_{2}^{\prime k}$ and $r_{8}^{\prime k}$ and the geometrical parameters to the joint values $\theta_{2}^{k}$ and $\theta_{8}^{k}$. All calculation details of the expressions $U, V$ and $W$ are given in Appendix A.

$$
\begin{array}{r}
\theta_{2}^{k}=V\left(r_{2}^{\prime k}, \kappa_{2}, \lambda_{2}, l_{12}, l_{32}\right) \\
\theta_{8}^{k}=W\left(r_{8}^{\prime k}, \kappa_{8}, \lambda_{8}, l_{18}, l_{38}\right)
\end{array}
$$

The vector of the actuated joints is thus:

$$
\mathbf{q}^{k}=\left[\begin{array}{llllll}
\theta_{1}^{k} & {r_{2}^{\prime}}^{k} & r_{8}^{\prime k} & \theta_{4}^{k} & \theta_{5}^{k} & \theta_{7}^{k}
\end{array}\right]^{T} .
$$

Using the relations (42), (43), (44), (45) and (46), the Forward Geometrical Model $f(1)$ and the Inverse Geometrical Model $g(6)$ are obtained. These relations link the controlled pose ${ }^{0} \mathbf{S}_{E}^{k}$ to the $(6 \times 1)$ vector of the actuated joint values $\mathbf{q}^{k}$ and the vector of the geometrical parameters $\xi$ (including all slidercrank mechanisms' geometrical parameters) (48).

$$
\begin{array}{r}
\xi=\left[\begin{array}{lllllllllllllllllllll}
\alpha_{1} & d_{1} & r_{1} & \alpha_{2} & d_{2} & r_{2} & \kappa_{2} & \lambda_{2} & l_{12} & l_{32} & \beta_{3} & \alpha_{3} & d_{3} \\
r_{3} & \alpha_{4} & d_{4} & r_{4} & \alpha_{5} & d_{5} & r_{5} & \alpha_{6} & d_{6} & r_{6} & \alpha_{7} & d_{7} & r_{7} \\
\beta_{E} & \alpha_{E} & d_{E} & r_{E} & \kappa_{8} & \lambda_{8} & l_{18} & l_{38} & d_{9} & d_{10} & d_{11}
\end{array}\right]^{T}
\end{array}
$$

\subsubsection{Error model and sensitivity analysis}

The geometrical error model is derived by calculating for each pose ${ }^{0} \mathbf{S}_{E}^{k}$, the Jacobian matrix of all parameters ${ }^{0} \mathbf{J}_{E}^{k}$ linking the positioning error ${ }^{0} d \mathbf{S}_{E}^{k}$ to the vector of the geometrical errors $d \xi$ according to the relation:

$$
{ }^{0} d \mathbf{S}_{E}^{k}={ }^{0} \mathbf{J}_{E}^{k} d \xi
$$


The columns of ${ }^{0} \mathbf{J}_{E}^{k}$ are computed by considering the variation of each geometrical parameter involved in the homogeneous matrices ${ }^{a(j)} \mathbf{T}_{j}$ used to describe the kinematic chain of Fig. 6. As a result ${ }^{0} \mathbf{J}_{E}^{k}$ can be viewed as the concatenation of the following submatrices:

$$
{ }^{0} \mathbf{J}_{E}^{k}=\left[{ }^{0} \mathbf{J}_{1, E}^{k}{ }^{0} \mathbf{J}_{2, E}^{k}{ }^{0} \mathbf{J}_{3, E}^{k}{ }^{0} \mathbf{J}_{4, E}^{k}{ }^{0} \mathbf{J}_{5, E}^{k}{ }^{0} \mathbf{J}_{6, E}^{k}{ }^{0} \mathbf{J}_{7, E}^{k}{ }^{0} \mathbf{J}_{E, E}^{k}\right] .
$$

${ }^{0} \mathbf{J}_{j, E}^{k}$ are the sub-matrices related to the transformations ${ }^{a(j)} \mathbf{T}_{j}$ (for $j=$ $\{1,2,3,4,5,6,7, E\})$ and their columns are calculated based on the relation (41) according to the method developed in [54]. A parameter $d \beta_{3}$ is introduced to describe the small orientation errors between the joint axes 2 and $3 . \quad d \beta_{3}$ represents the possible orientation errors of the whole four bar linkage with respect to the open kinematic chain [9]. Moreover, the relations (43), (44), (45), and (46) have to be considered since the geometrical parameters they depend of can also be affected by some errors. Since these errors decrease the robot pose accuracy, they have to be included in the vector $d \xi$ and their related columns have to be added to the Jacobian matrix ${ }^{0} \mathbf{J}_{E}^{k}$. This is done by differentiating those relations with respect to the geometrical parameters. This leads to:

$$
\begin{aligned}
& d \theta_{5}=-d \theta_{6}, \\
& d \theta_{3}=a_{3}^{k}\left(d \theta_{2}-d \theta_{8}\right)+b_{3}^{k} d d_{3}+c_{3}^{k} d d_{9}+d_{3}^{k} d d_{10}+e_{3}^{k} d d_{11}, \\
& d \theta_{2}=a_{2}^{k} d r_{2}^{\prime}+b_{2}^{k} d \kappa_{2}+c_{2}^{k} d \lambda_{2}+d_{2}^{k} d l_{12}+e_{2}^{k} d l_{32}, \\
& d \theta_{8}=a_{8}^{k} d r_{8}^{\prime}+b_{8}^{k} d \kappa_{8}+c_{8}^{k} d \lambda_{8}+d_{8}^{k} d l_{18}+e_{8}^{k} d l_{38} .
\end{aligned}
$$

The analytical values of $a_{m}^{k}, b_{m}^{k}, c_{m}^{k}, d_{m}^{k}$ and $e_{m}^{k}$ (for $m=\{2,3,8\}$ ) are obtained using symbolic Matlab Software and are merged into the expression of ${ }^{0} \mathbf{J}_{E}^{k}$ in (52). The resulting formulation of ${ }^{0} \mathbf{J}_{E}^{\prime k}$ can be written as follows:

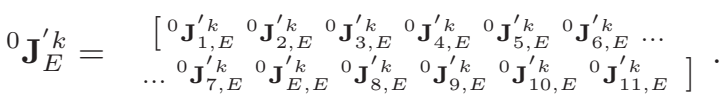

Details of ${ }^{0} \mathbf{J}_{E}^{\prime} k$ submatrices are given in Appendix B. As a result, for each configuration $k$, the calculation of the $6 \times 43$ matrix ${ }^{0} \mathbf{J}_{E}^{\prime} k$ allows to calculate according to (49) the effects of the 43 geometrical errors $d \xi$ on the pose error ${ }^{0} d \mathbf{S}_{E}^{k}$. All geometrical errors are gathered in the vector $d \xi$ defined in (53). This resulting linear model is used for both sensitivity/observability study and linear identification of the geometrical errors.

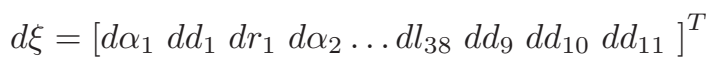

For a geometrical error $d \xi_{j}$ of vector $d \xi$ and a robot configuration $k$, two sensitivity indexes $\mu_{P_{j}}^{k}$ and $\mu_{\Phi_{j}}^{k}$ can be defined as the norm of the column $j$ of respectively the Jacobian matrices of position and orientation ${ }^{0} \mathbf{J}_{\mathbf{P}}{ }_{E, j} k$ and ${ }^{0} \mathbf{J}_{\phi E, j}{ }^{\prime k}$ that are defined according to:

$$
{ }^{0} \mathbf{J}_{E, j}^{\prime k}=\left[\begin{array}{c}
0 \\
{ }^{0} \mathbf{J}_{\mathbf{P}_{j, E}{ }^{\prime k}} \\
{ }^{0} \mathbf{J}_{\phi_{j, E}{ }^{\prime}}
\end{array}\right] .
$$

The values of the two indexes are calculated according to the relations [55]: 


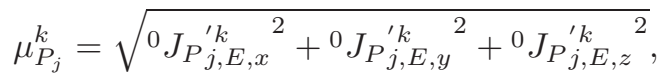

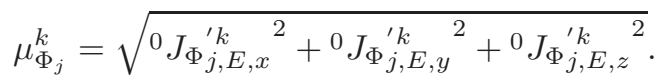

For the robot configuration $k$ the indexes link the norm of the position and orientation errors ${ }^{0} d \mathbf{P}_{j, E}^{\prime k}$ and ${ }^{0} d \boldsymbol{\Phi}_{j, E}^{\prime k}$ to the geometrical error value $d \xi_{j}$ according to:

$$
\begin{aligned}
& \left\|{ }^{0} d \mathbf{P}_{j, E}^{\prime k}\right\|=d \xi_{j} \sqrt{{ }^{0} J_{P}^{\prime k, E, x}{ }^{2}+{ }^{0} J_{P}{ }_{j, E, y}{ }^{2}{ }^{2}+{ }^{0} J_{P}{ }_{j, E, z}{ }^{2}}=\mu_{P_{j}}^{k} d \xi_{j},
\end{aligned}
$$

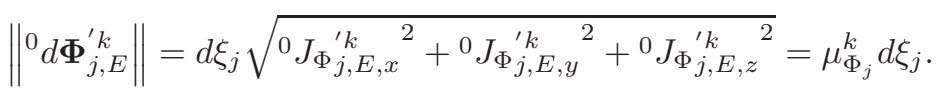

All indexe's values are derived by calculating the Jacobian matrices [42] ${ }^{0} \mathbf{J}_{\mathbf{P} E, j}{ }^{\prime k}$ and ${ }^{0} \mathbf{J}_{\phi E, j}{ }^{\prime k}$ for 27 robot configurations corresponding to uniformly distributed poses in the work volume defined by (59) and shown in Fig. C.20 (Appendix C):

$$
1.4 \leq{ }^{0} P_{E, x}^{k} \leq 2.1 m, \quad-0.3 \leq{ }^{0} P_{E, y}^{k} \leq 0.3 m, \quad 1.4 \leq{ }^{0} P_{E, z}^{k} \leq 1.7 m
$$

For each geometrical error $d \xi_{j}$, the maximum and the mean values of all indexes are given in Fig. C.21, C.22 for the position error and C.23, C.24 of the orientation error (Appendix C). Results show that the geometrical errors of the four bar linkage and the slider-crank mechanisms have the most important influence onto the position and orientation errors of the end-effector.

\subsubsection{Geometrical identification}

Using the linear error model derived in 3.2.2, the parameters observability study is performed by studying the norm of the columns and the conditioning of the global jacobian matrix ${ }^{0} \mathbf{J}_{7}^{G}$ [14]. This allows to select the vector $d \xi^{B}$ of the geometrical errors that can be identified independently. For the 27 robot configurations corresponding to the poses described by (59) the observability study shows that 6 parameters $d \kappa_{8}, d \kappa_{2}, d r_{8}^{\prime}, d d_{9}, d r_{2}$ and $d r_{7}$ cannot be identified independently of other parameters of $d \xi$. Therefore, they are removed from vector $d \xi$ to define $d \xi^{B}$. The linear combinations existing between $d \xi^{B}$ and the set of parameters $d \kappa_{8}, d \kappa_{2}, d r^{\prime}{ }_{8}, d d_{9}, d r_{2}, d r_{7}$ are given in Appendix C. They are due to the fact some of these errors act along or around parallel or collinear axes and therefore their effects onto the position and/or orientation of frame $R_{7}$ cannot be decoupled.

The 37 geometrical errors of $d \xi^{B}$ are then identified iteratively using the 27 measurements of the position and orientation of frame $R_{7}$ achieved using the NIKON-METROLOGY K600-10 system (See section 2.1.5). The values of identified geometrical errors are given in Appendix C. The figure 7 gives the norm of both position and orientation residual pose errors vector for each iteration. The resulting pose accuracy after calibration is tested using 16 poses different from the 27 used for identification and uniformly distributed the workspace defined by (59). This verification shows that the final pose error is reduced from $0.96 \mathrm{~mm}$ and $0.14 \mathrm{deg}$ to $0.24 \mathrm{~mm}$ in position and $0.03 \mathrm{deg}$ in orientation in the work volume considered. The geometrical calibration allows to improve the static pose accuracy about $75 \%$ in both position and orientation. 

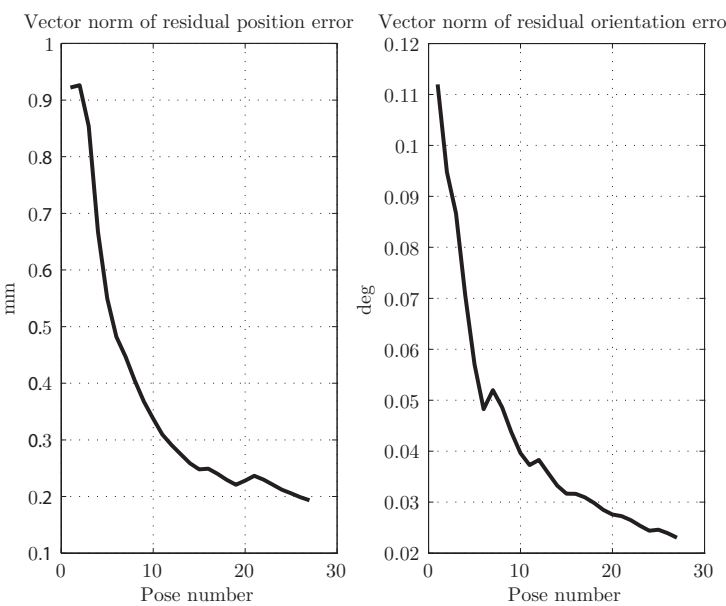

Figure 7: Residual pose error obtained during iterative identification of geometrical errors

\subsection{Elastic calibration of the KUKA-IR663}

\subsubsection{Calibration of the KUKA-IR663 parametric elastic model}

Derivation of the parametric elastic model. In order to derive the stiffness model of the KUKA, all links of the closed-loop kinematic chain are considered as beams and nodes. The method that has been presented in the first section is developed to derive in an analytical and systematic way, the stiffness matrix of the structure. The assumptions we made to derive the stiffness model of the KUKA's structure are the following:

- The beam theory is applicable to all links.

- The wrist is stiff enough to be neglected in the stiffness modeling (it is considered as a rigid body).

- The structure is modeled in the plane but can be excited in the three dimensions. It can be extended to a $3 \mathrm{D}$ model by applying a solicitation on the TCP along $\mathbf{y}_{0}$ axis.

For the elastic modeling, all nodes of the robot have been labeled (Fig. 8). The compliance of the joints has been integrated in the model to fully describe the elastic behavior of the closed-loop kinematic chain. Based on the labels used in Fig. 8, all the stiffness matrices of the beams and joints are expressed within the reference frame $R_{0}$ and are then mapped into the global stiffness matrix of the robot structure ${ }^{0} \mathbf{K}_{G}$ (21). Boundary conditions are introduced to express the fact that the robot is embedded in the ground at node 0 . These conditions are expressed by the equation system:

$$
\left\{\begin{array}{l}
{ }^{0} d \mathbf{P}_{0}=\mathbf{0}_{\mathbf{3}, 1} \\
{ }^{0} d \mathbf{\Phi}_{0}=\mathbf{0}_{\mathbf{3}, \mathbf{1}}
\end{array} .\right.
$$

To integrate these conditions, the size of the global stiffness matrix ${ }^{0} \mathbf{K}_{\mathbf{G}}$ is reduced by deleting the rows and the columns corresponding to the blocked 


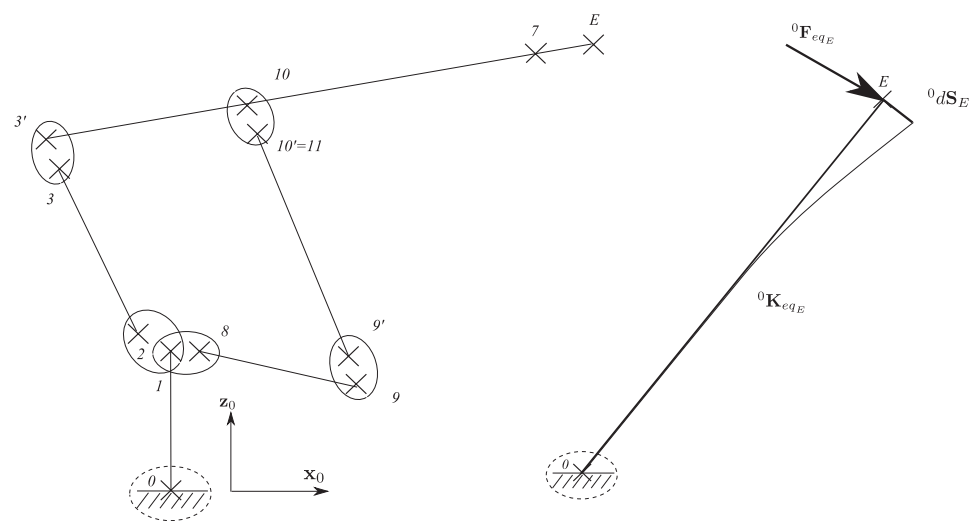

Figure 8: Beam model of the KUKA-IR663 structure

displacements. The dimension of the reduced stiffness matrix ${ }^{0} \mathbf{K}_{\mathbf{C}}$ is $(60 \times 60)$ (61).

$$
\begin{aligned}
& { }^{0} \mathbf{K}_{C}=
\end{aligned}
$$

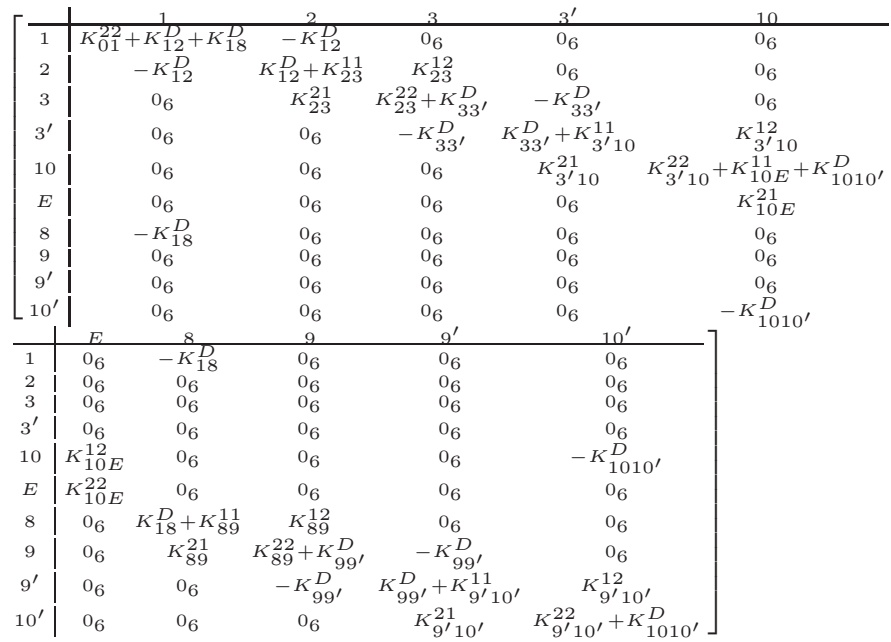

As the boundary conditions are perfectly defined, so as to prevent any possible structure's displacements, the stiffness matrix is symmetric positive definite and therefore invertible.

The vectors ${ }^{0} \mathbf{F}_{C}$ and ${ }^{0} d \mathbf{S}_{C}$ that describe the nodal wrenches and displacements of the structure are expressed within $R_{0}$ as follows:

$$
\begin{gathered}
{ }^{0} \mathbf{F}_{C}=\left[\begin{array}{lllll}
{ }^{0} \mathbf{F}_{1}^{T} & { }^{0} \mathbf{F}_{2}^{T} & \ldots & { }^{0} \mathbf{F}_{9^{\prime}}^{T} & { }^{0} \mathbf{F}_{10^{\prime}}^{T}
\end{array}\right]^{T}, \\
{ }^{0} d \mathbf{S}_{C}=\left[\begin{array}{lllll}
{ }^{0} d \mathbf{S}_{1}^{T} & { }^{0} d \mathbf{S}_{2}^{T} & \ldots & { }^{0} d \mathbf{S}_{9^{\prime}}^{T} & { }^{0} d \mathbf{S}_{10^{\prime}}^{T}
\end{array}\right]^{T} .
\end{gathered}
$$

From the relation (23), the positioning error ${ }^{0} d \mathbf{S}_{E}$ of the end-effector linked to the elastic deformations of the structure can be calculated as a function of ${ }^{0} \mathbf{F}_{C}$ (function of the wrench ${ }^{0} \mathbf{F}_{E}$ applied on the TCP during the process) and 
from the joint configuration $\mathbf{q}^{k^{*}}=\left[\begin{array}{ll}\theta_{2} & \theta_{8}\end{array}\right]^{T}$ (Fig. 8). For that purpose, the stiffness matrix, the nodal wrench and nodal displacements are partitioned as below:

$$
\left[\begin{array}{c|c}
{ }^{0} \mathbf{K}_{R, R} & { }^{0} \mathbf{K}_{R, E} \\
\hline{ }^{0} \mathbf{K}_{E, R} & { }^{0} \mathbf{K}_{E, E}
\end{array}\right]\left[\begin{array}{c}
{ }^{0} d \mathbf{S}_{R} \\
\hline{ }^{0} d \mathbf{S}_{E}
\end{array}\right]=\left[\frac{{ }^{0} \mathbf{F}_{R}}{{ }^{0} \mathbf{F}_{E}}\right]
$$

Rewriting the equation (62), following the expression of an equivalent parametric elastic model (26), the nodal displacement of the TCP can be calculated as follows:

$$
{ }^{0} d \mathbf{S}_{E}=\left[{ }^{0} \mathbf{K}_{e q_{E}}\right]^{-1}{ }^{0} \mathbf{F}_{e q_{E}}
$$

with:

$$
\begin{aligned}
{ }^{0} \mathbf{F}_{e q_{E}} & ={ }^{0} \mathbf{F}_{E}-{ }^{0} \mathbf{K}_{E, R}\left({ }^{0} \mathbf{K}_{R, R}\right){ }^{-1}{ }^{0} \mathbf{F}_{R}, \\
{ }^{0} \mathbf{K}_{e q_{E}} & ={ }^{0} \mathbf{K}_{E, E}-{ }^{0} \mathbf{K}_{E, R}\left({ }^{0} \mathbf{K}_{R, R}\right){ }^{-1}{ }^{0} \mathbf{K}_{R, E} .
\end{aligned}
$$

Elastic identification. The resulting Direct Elastic Model whose parameters have to be identified is described by the relation:

$$
{ }^{0} d \mathbf{S}_{E}=\left[\begin{array}{c}
{ }^{0} d \mathbf{P}_{E} \\
{ }^{0} d \boldsymbol{\Phi}_{E}
\end{array}\right]=h\left(\mathbf{q}^{k}, \xi,{ }^{0} \mathbf{F}_{e q}, \boldsymbol{\Lambda}, \boldsymbol{\Gamma}\right)=\left({ }^{0} \mathbf{K}_{e q}\right){ }^{-1}{ }^{0} \mathbf{F}_{e q} .
$$

The joint stiffness parameters are gathered in the vector $\boldsymbol{\Gamma}$. Since the joints 3, 9 and 10 are passive (Fig. 8), their axial rotational stiffness $K_{a r}$ is supposed being close to zero. For numerical problems, this value is set to $10^{-10}$ $N m . r a d^{-1}$. Since the structure is loaded in the plane $\left(O_{0}, \mathbf{x}_{\mathbf{0}}, \mathbf{z}_{\mathbf{0}}\right)$, the values of the translational axial stiffness $K_{a}$ and the rotational radial stiffness $K_{r r}$ of the joints cannot be identified using the loading cases and thus are set to high values (respectively $10^{15} \mathrm{~N} \cdot \mathrm{m}^{-1}$ and $10^{15} \mathrm{Nm} \cdot \mathrm{rad}^{-1}$ ) [43, 56]. To fully describe the elastic behavior of the closed-loop kinematic chain (Fig. 8), the $K_{a r}$ parameters for the active joints 2 and 8 , which stands for the axial rotational stiffness of the actuators, and the $K_{r}$ parameters for the joints 2, 3, 8, 9 and 10 have to be identified by using the robot's elastic pose error measurements.

The pose errors that are measured correspond to a range of payloads for different robot configurations. Due to the possible use of a FANUC S420iF available in the cell, the horizontal force vector is generated by deadweight suspended to the free-end of a cable guided by a pulley mounted on its endeffector (Fig. 5). Four loads of 30, 60, 90 and $110 \mathrm{~kg}$ were used to apply the horizontal force. The same loads were also applied directly to the end-effector in order to create a vertical payload. The poses for the identification are selected from a regular grid of $k=63$ poses included in a vertical plane (65).

$$
1.25 \leq{ }^{0} P_{E, x}^{k} \leq 2.25 m \quad 0.85 \leq{ }^{0} P_{E, z}^{k} \leq 2.15 m \quad{ }^{0} P_{E, y}^{k}=0 .
$$

Therefore the TCP is moved to each of the 63 poses for which position and orientation measurements are done. For each pose, data acquisition is performed with the K600-10 system during $5 s$ with a $20 \mathrm{~ms}$ sampling rate. In order to minimize the effects of measurement noise after pose stabilization, we considered the mean of 100 measurement points as the measurand value. The statistic average of the distance differences is about $35 \mu \mathrm{m}$ per 3D-point with 
a standard deviation about $80 \mu \mathrm{m}$ and a maximum difference of $190 \mu \mathrm{m}$. The position accuracy of the K600-10 system for a single point is up to $37 \mu \mathrm{m}$.

The elastic displacements have been obtained by the substraction of the poses measured with and without payload. The error ${ }^{0} E_{E}^{k}$, merged into a cost function (67), is used to identify the structure elastic model is:

$$
{ }^{0} E_{E}^{k}=\left\|{ }^{0} d \mathbf{P}_{E}^{m, k}-{ }^{0} d \mathbf{P}_{E}^{k}\right\| .
$$

Note that only the pose position error is used for the calculation of ${ }^{0} E_{E}^{k}$ but the orientation error could also have been involved for accuracy purpose. The norms of the measured elastic displacements within the plane $\left(O_{0}, \mathbf{x}_{0}, \mathbf{z}_{0}\right)$ are depicted by Fig. 9. For distant configurations from the vertical axis $\mathbf{z}_{0}$, the elastic displacements are important. The norm ranges from $1.6 \mathrm{~mm}$ to $2.5 \mathrm{~mm}$ for a vertical load of $1100 \mathrm{~N}$. Complementary figures of the TCP measured elastic displacements are given in Appendix D. Furthermore, horizontal loads induce substantial vertical TCP deflections for low heights $(0.9 \mathrm{~mm}$ for a 1100 $N$ load).

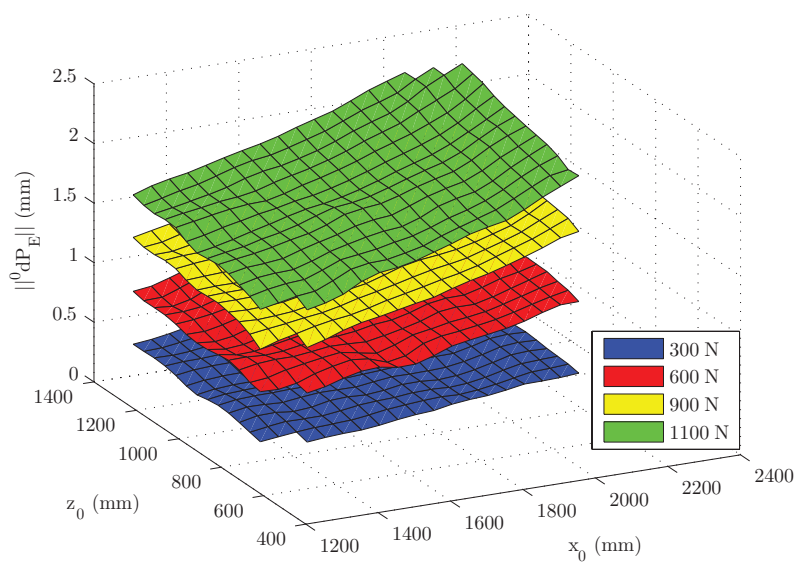

Figure 9: Norm of the measured TCP's elastic displacements for vertical static loads of 300, 600,900 and $1100 \mathrm{~N}$

The numerical values of the joint stiffness gathered in the vector $\boldsymbol{\Gamma}$ are identified by minimizing the quadratic criterium (67) for a set of $n_{m}$ pose and load configurations:

$$
C(\boldsymbol{\Gamma})=\frac{1}{n_{m}} \sum_{k=1}^{n_{m}}\left({ }^{0} E_{E}^{k}\right)^{2} .
$$

From a total of 8 measurement sets (4 loads along $\mathbf{z}_{\mathbf{0}}$ and 4 along $\mathbf{x}_{\mathbf{0}}$ directions), the two sets of elastic displacements measured for the $900 \mathrm{~N}$ load are selected for the identification. This choice has been done to minimize the influence of measurement noise (very important for low payloads) and to fit the considered robot applications (assembly, forming, machining) that require a high level of load. A total of $n_{m}=126$ data configurations were used for the identification which can be achieved either with traditional methods (Newton, 

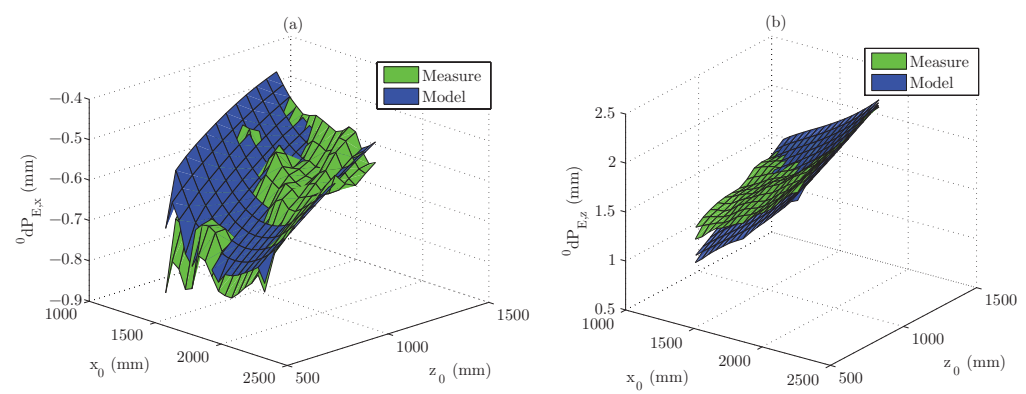

Figure 10: Experimental and parametric model validation of data set: (a) horizontal elastic displacement for a $600 \mathrm{~N}$ horizontal load (b) vertical elastic displacement for a $1100 \mathrm{~N}$ vertical load

Levenberg-Marquardt) or with stochastic methods. Nevertheless, the genetic algorithms find here all their utility considering the difficulties to obtain an initial value of the joint stiffness (from the manufacturers data). To identify the stiffness parameters an optimization procedure is implemented in modeFRON$\mathrm{TIER}^{2}$, a multi-objective optimization software. An object-oriented programming language, MATLAB 2008b, is used for the parametric elastic modeling. In order to minimize the difference between numerical and experimental results for the $900 \mathrm{~N}$ load, a genetic optimization algorithm is applied to automatically launch the parametric elastic model and minimize the criterium described by (67) $[57]$.

The identified stiffness values (Tab. 3) obtained with an optimum design have been used to evaluate the elastic displacements of the manipulator for the other sets of loads (Fig. 10). The identified elastic model is appropriate for the calculation of the manipulator TCP deflections under an external load. Moreover, the elastic behavior of the manipulator is linear over the range of load (Fig. 9). Another fact is the good fitting of the elastic displacement when the wrist is far from the vertical axis of the base frame $R_{0}$. On the contrary, when the end-effector is moved close to $\mathbf{z}_{0}$ axis, the model is too stiff and does not fit anymore with the experimental data. The error exceeds $15 \%$ on the vertical elastic displacements for a $1100 N$ vertical load (Fig. 10-(b)). This error may come from the assumption made on the modeling of the two slidercrank mechanisms. The stiffness of the two slider-crank mechanisms has been described by an equivalent rotational stiffness located at the center of the joints $(1,2)$ and $(1,8)$. A more realistic model should integrate each ball screw using extra beams connected to robot links 2 and 8 on one hand and to the first link through prismatic and revolute joints on the other hand (Fig. A.19). The stiffness of the actuators will thus be reported on the prismatic joints of the ball screws.

The Fig. 11, obtained through a numerical approach, shows the beam deformations represent about $10 \%$ of the overall elastic displacements of the TCP. This can be easily understood considering the design of the robot's links which gives them a high level of stiffness. Note on Fig. 11-(b) that the vertical defor-

\footnotetext{
${ }^{2}$ http://www.esteco.com/home/mode_frontier
} 

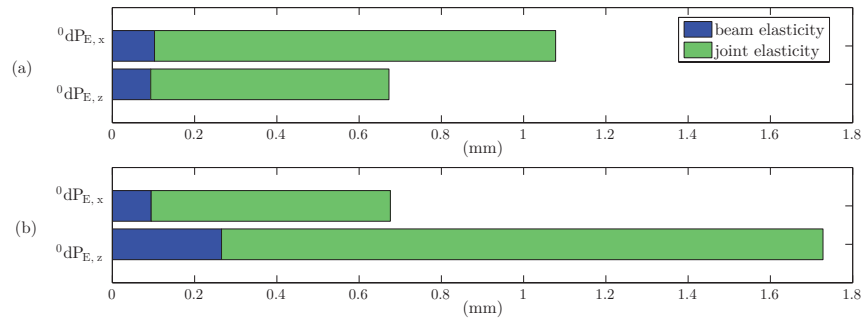

Figure 11: Influence of the beam and joint deformations on the overall TCP elastic displacements at the position $P_{E, x}=1815 \mathrm{~mm}$ and $P_{E, z}=910 \mathrm{~mm}$ for an horizontal static load of $900 \mathrm{~N}$ (a) and for a vertical static load of $900 \mathrm{~N}$ (b)

\begin{tabular}{|c|c|c|c|c|}
\hline & \multicolumn{3}{|c|}{ Complete Model } & Reduced Model \\
\hline$\Gamma \backslash$ Design & Design 1 & Design 2 & Design 3 & \\
\hline$K_{a r}^{12}\left(N m \cdot r a d^{-1}\right)$ & $1.72 .10^{9}$ & $1.72 .10^{9}$ & $1.72 .10^{9}$ & $1.72 .10^{9}$ \\
\hline$K_{a r}^{18}\left(N m \cdot r a d^{-1}\right)$ & $1.56 .10^{9}$ & $1.56 .10^{9}$ & $1.56 .10^{9}$ & $1.56 .10^{9}$ \\
\hline$K_{r}^{12}\left(N \cdot m^{-1}\right)$ & $5.27 .10^{10}$ & $2.32 .10^{10}$ & $6.22 .10^{10}$ & 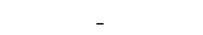 \\
\hline$K_{r}^{18}\left(N \cdot m^{-1}\right)$ & $2.25 .10^{8}$ & $7.92 .10^{9}$ & $2.22 .10^{9}$ & - \\
\hline$K_{r}^{33^{\prime}}\left(N \cdot m^{-1}\right)$ & $5.10 .10^{9}$ & $7.1 .10^{10}$ & $3.68 .10^{10}$ & - \\
\hline$K_{r}^{99^{\prime}}\left(N \cdot m^{-1}\right)$ & $6.63 .10^{5}$ & $3.63 .10^{9}$ & $1.56 .10^{6}$ & - \\
\hline$K_{r}^{1010^{\prime}}\left(N \cdot m^{-1}\right)$ & $6.12 .10^{4}$ & $5.56 .10^{4}$ & $6.11 .10^{4}$ & - \\
\hline$K_{r e q}^{12}\left(N \cdot m^{-1}\right)$ & $4.65 .10^{9}$ & $1.75 .10^{10}$ & $2.31 .10^{10}$ & $8.32 .10^{9}$ \\
\hline$K_{\text {req }}^{18}\left(N \cdot m^{-1}\right)$ & $5.59 .10^{4}$ & $5.55 .10^{4}$ & $5.56 .10^{4}$ & $5.56 .10^{4}$ \\
\hline$\overline{C(\boldsymbol{\Gamma})}$ & $\overline{0.92}$ & $\overline{0.92}$ & $\overline{0.92}$ & $\overline{0.92}$ \\
\hline
\end{tabular}

Table 3: Identified values of the joint stiffness parameters

mation due to the beam stiffness is more important for a vertical load. This is due to the flexion of the main arm corresponding to the beam $(10,7)$.

This remark shows that the elastic modeling of the robot's structure could have also been achieved by simplifying our complete model into a parametric lumped model [55]. Nevertheless, for applications requiring a high level of accuracy (assembly, Friction Steer Welding, machining, etc.) all elastic effects that degrade the robot's pose accuracy (joints, bodies, robot support frame) have to be described and compensated. We think this systematic approach of the parametric beam model is more suitable since all stiffness elements can be modeled which may lead to an interpretation of all effects.

Whatever parametric approach is used (beam models or lumped), some important problems remain:

- The first concerns the coupling between some stiffness elements. In some robot's configurations, the stiffness of certain joints or beams can be replaced by serial or parallel equivalent stiffness elements. This leads to singularities in the elastic model since some columns of the stiffness matrix ${ }^{0} \mathbf{K}_{C}$ are dependent the ones from the others and thus the matrix is not full ranked. For the KUKA-IR663, the radial stiffness values of joints corresponding to the nodes $(1,2)$ and $\left(3,3^{\prime}\right)$ on one side and to the nodes $(1,8),\left(9,9^{\prime}\right)$ and $\left(10,10^{\prime}\right)$ on the other side of the closed loop can be viewed as springs in serial and therefore they can be replaced by two equivalent radial stiffness values $K_{r e q}^{12}$ and $K_{r e q}^{18}$ such as:

$$
\begin{aligned}
& \frac{1}{K_{r e q}^{12}}=\frac{1}{K_{r}^{12}}+\frac{1}{K_{r}^{33^{\prime}}}, \\
& \frac{1}{K_{r e q}^{18}}=\frac{1}{K_{r}^{18}}+\frac{1}{K_{r}^{99^{\prime}}}+\frac{1}{K_{r}^{1010^{\prime}}} .
\end{aligned}
$$


For the identification of the stiffness values, an infinity of solutions for $K_{r}^{12}, K_{r}^{18}, K_{r}^{33^{\prime}}, K_{r}^{99^{\prime}}$ and $K_{r}^{1010^{\prime}}$ are possible. One can see that different sets of these joint-stiffness' values lead to a minimum of the quadratic index as shown in Table 3. The resulting joint-stiffness parameters are not independent and thus the global solution does not depict the physical stiffness elements of the real structure.

- Some stiffness effects cannot be easily described and identified, since they are non-linearly dependent of the robot joint configurations, the external load, the temperature, etc. For the KUKA-IR663, this occurs if one considers the stiffness of the slider-crank mechanisms driving the revolute joints $(1,2)$ and $(1,8)$ of Fig. 8.

- The elastic modeling can be complex and time consuming to establish for complex structures with closed loops (parallel robots and machines).

In these cases, an interesting alternative approach to the previously presented parametric method is to perform the elastic modeling using fuzzy inference systems. Next section gives an illustration of this fuzzy logic modeling with experimental results for the elastic calibration of the KUKA-IR663.

\subsubsection{Fuzzy Model Identification}

The identification method detailed in section 2.2.2 processes the experimental data obtained with the K600-10 system to create a fuzzy inference system of the elastic behavior of the KUKA-IR663. The KUKA's structure is only modeled in the plane $\left(O_{0}, \mathbf{x}_{\mathbf{0}}, \mathbf{z}_{\mathbf{0}}\right)$ as in the elastic parametric modeling. The fuzzy logic model development is driven by measurement data and without any priori assumption made on the possible elastic sources.

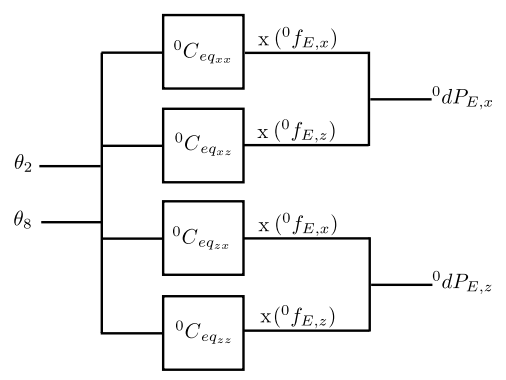

Figure 12: Input and output variables for FL model

The fuzzy inference system is designed with four parallelized single output FL models (Fig. 12). Each sub-FL model predicts a component of the equivalent compliance matrix of the manipulator. Inputs from the FL model are the joint values $\theta_{2}$ and $\theta_{8}$. The TCP's translational elastic displacements in the plane $\left(O_{0}, \mathbf{x}_{\mathbf{0}}, \mathbf{z}_{\mathbf{0}}\right),{ }^{0} d P_{E, x}$ and ${ }^{0} d P_{E, z}$ are obtained by multiplying the identified compliance matrix's components with the horizontal and vertical forces ${ }^{0} f_{E, x}$ and ${ }^{0} f_{E, z}$ by relation (70). So it is assumed that the elastic displacements are linear with respect to external load.

$$
\left\{\begin{array}{l}
{ }^{0} d P_{E, x}={ }^{0} C_{e q_{x x}}{ }^{0} f_{E, x}+{ }^{0} C_{e q_{x z}}{ }^{0} f_{E, z} \\
{ }^{0} d P_{E, z}={ }^{0} C_{e q_{z x}} f_{E, x}+{ }^{0} C_{e q_{z z}} f_{E, z}
\end{array}\right.
$$


The FL model is entirely identified from the experimental data, since a sufficiently rich database has been obtained using the K600-10 system. The 900 $N$ payload subset of measurements is selected to create training and test sets. Among the 126 poses of the grid for the $900 \mathrm{~N}$ payload 36 measurements points are selected randomly to construct the test set $\mathcal{T}$. The 90 remaining points are the learning set $\mathcal{A}$.
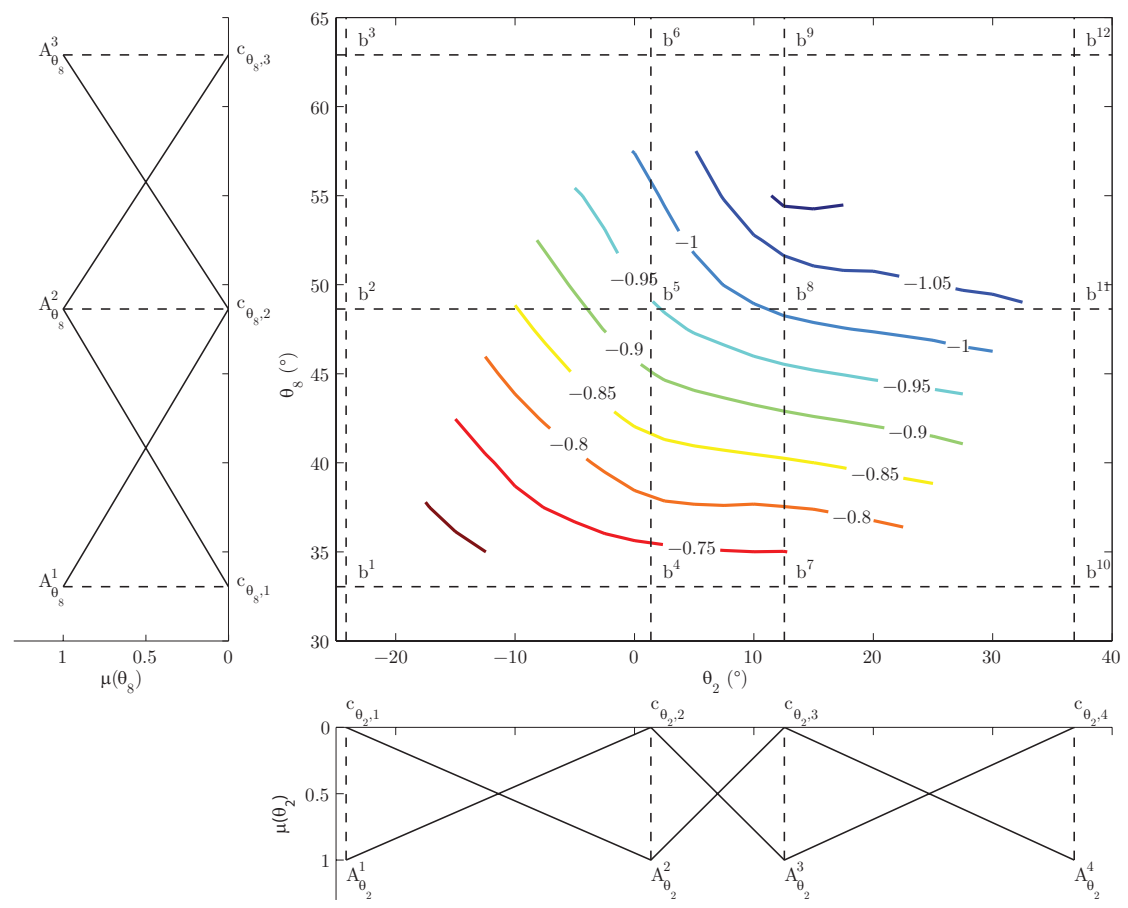

Figure 13: Fuzzy Logic model of the compliant coefficient ${ }^{0} C_{e q_{x x}}$ and Membership Functions for the model variables $\theta_{2}$ and $\theta_{8}$

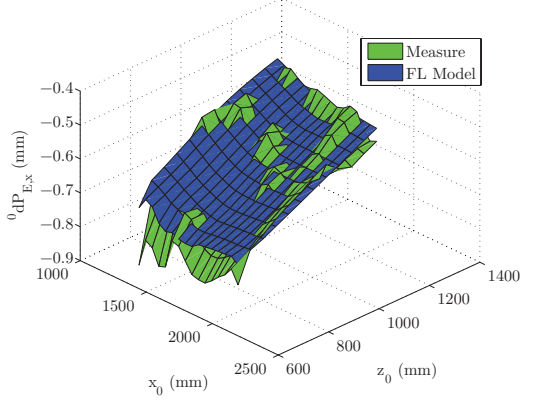

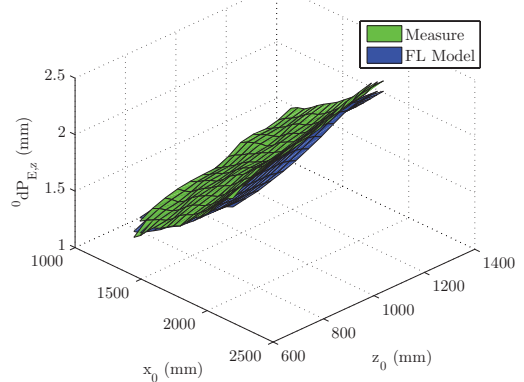

Figure 14: Experimental and Fuzzy Logic model validation of data set: (a) horizontal elastic displacement for a $600 \mathrm{~N}$ horizontal load (b) vertical elastic displacement for a $1100 \mathrm{~N}$ vertical load

An initial FL model is created with two MFs per input to begin the iter- 
ative identification procedure. Using Solis and Wets algorithm a sub-optimal solution of the modal values, i.e. the placement of the MFs, is obtained. The conclusions of the rules are modified by a Gradient Descent method to establish appropriate correlation between the measured and calculated TCP poses. After few iterations, the use of four and three MFs for $\theta_{2}$ and $\theta_{8}$, respectively, allows to obtain the best compromise for the prediction of the compliance behavior of the KUKA-IR663. So a total of 12 rules are used in each single output model. The aggregated output of a single FL model is a weighted average of the contribution of the 12 rules according to (36).

Table 4 gives the modal values defining the fuzzy sets and the conclusions of the rules for the compliance coefficient ${ }^{0} C_{e q_{x x}}$. Fig. 13 shows the surface that represents the compliance coefficient ${ }^{0} C_{e q_{x x}}$, over the input domain, and the associated MFs for the input variables $\theta_{2}$ and $\theta_{8}$. The shape of the surface is regular and shows a nonlinear change of the structure's compliance with a variation of either $\theta_{2}$ or $\theta_{8}$.

Table 4: Rule base of the Fuzzy Logic Model of the compliant coefficient ${ }^{0} C_{e q_{x x}}$

\begin{tabular}{|c|c|c|c|}
\hline Rule $i$ & $c_{\theta_{2}, m_{\theta_{2}}}(\mathrm{deg})$ & $c_{\theta_{8}, m_{\theta_{8}}}(\mathrm{deg})$ & $b^{i}(\mathrm{~mm})$ \\
\hline 1 & -24.15 & 33.04 & -0.639 \\
2 & -24.15 & 48.63 & -0.718 \\
3 & -24.15 & 62.90 & -0.910 \\
4 & 1.37 & 33.04 & -0.730 \\
5 & 1.37 & 48.63 & -0.953 \\
6 & 1.37 & 62.90 & -1.040 \\
7 & 12.54 & 33.04 & -0.711 \\
8 & 12.54 & 48.63 & -1.010 \\
9 & 12.54 & 62.90 & -1.218 \\
10 & 36.83 & 33.04 & -0.769 \\
11 & 36.83 & 48.63 & -1.057 \\
12 & 36.83 & 62.90 & -1.156 \\
\hline
\end{tabular}

In order to verify the global FL model accuracy, the TCP elastic displacements that this model allows to predict are compared with those measured experimentally. This is done by using two other sets of measurements performed with loads of 600 and $1100 \mathrm{~N}$ that are not already used for the training of the FL model. As one can see on Fig. 14, the pose errors induced by the elastic effects which are predicted by the FL model do fit the experimental results. The values of the RMS errors, for the 600 and the $1100 N$ loads, given in table (5) are about 52 and 84 microns respectively. Their magnitude can be regarded as minor compared with the measurement system accuracy.

\begin{tabular}{|c||c|c|c|c|c|}
\hline Method & Load & RMS $(\mathrm{mm})$ & $\begin{array}{c}\text { Max error } \\
\text { norm }(\mathrm{mm})\end{array}$ & $\begin{array}{c}\text { Max error } \\
\mathbf{x}_{\mathbf{0}}(\mathrm{mm})\end{array}$ & $\begin{array}{c}\text { Max error } \\
\mathbf{z}_{0}(\mathrm{~mm})\end{array}$ \\
\hline \hline \multirow{4}{*}{ FL } & 300 & 0.064 & 0.138 & 0.127 & 0.080 \\
& 600 & 0.052 & 0.160 & 0.159 & 0.076 \\
& 900 & 0.057 & 0.144 & 0.114 & 0.121 \\
& 1100 & 0.084 & 0.183 & 0.145 & 0.175 \\
\cline { 2 - 7 } & all & 0.065 & - & - & - \\
\hline \hline \multirow{5}{*}{ Parametric } & 300 & 0.082 & 0.142 & 0.140 & 0.079 \\
& 600 & 0.093 & 0.184 & 0.166 & 0.167 \\
& 900 & 0.122 & 0.282 & 0.252 & 0.271 \\
& 1100 & 0.136 & 0.371 & 0.196 & 0.351 \\
\hline & all & 0.110 & - & - & - \\
\hline
\end{tabular}

Table 5: Comparison of the elastic parametric and fuzzy logic modeling methods 

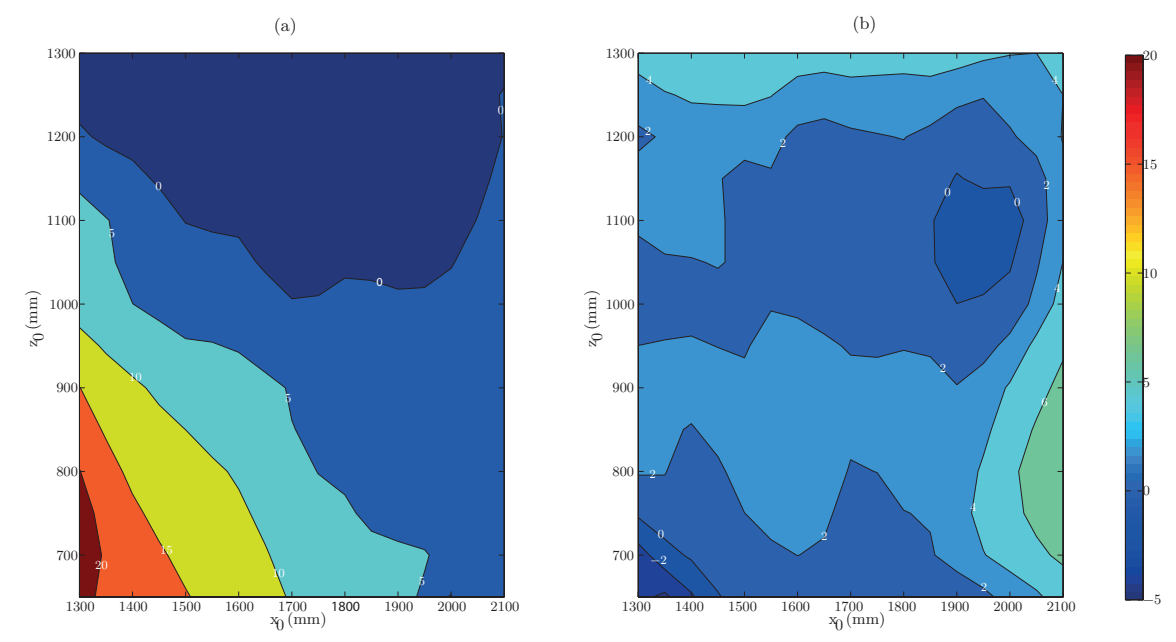

Figure 15: Error in percent on the vertical displacement for a $1100 \mathrm{~N}$ load: (a) parametric modeling (b) fuzzy logic modeling

\subsubsection{Discussion}

Compared to the results obtained with the elastic parametric model, the FL model allows to get a better accuracy. The RMS errors, considering all sets of loads, is about $65 \mu \mathrm{m}$. With the parametric approach the RMS is $110 \mu \mathrm{m}$. Moreover, Table (5) shows that the FL model prediction of pose errors is more robust to the load changes. Indeed in the case of the 900 and $1100 \mathrm{~N}$ loads, the RMS error are 57 and $84 \mu m$ respectively with the FL modeling and about 122 and $136 \mu \mathrm{m}$ respectively with the parametric approach. Moreover, maximum errors are about 144 and 183 against 282 and $371 \mu m$ respectively. As we explained before, this must be due to the fact that the parametric model does not take into account the effects of all possible elastic sources which non-linear behavior degrades the pose accuracy. For example the parametric model does not consider the compliance induced by robot slider-crank mechanisms whereas the FL in its global approach allows to compensate these lacks of modeling. The accuracy of the FL model is also described by Fig (15) where one can see that the errors are predicted uniformly within the robot workspace. This figure depicts the error in percent between the measurements and the elastic models.

Simulation and experimental results show the effectiveness of the proposed identified FL model under various loading conditions. The proposed modeling and identification method uses the advantage of fuzzy logic such as simplicity, rapidity and robustness. Hence, the fuzzy modeling approach has great potential in developing accurate prediction models and in improving the understanding of structure's characteristics. The KUKA IR-663 illustrative example, given in this paper, shows that the proposed method is general and thus useful. To extend the validity of the method, the elasto-geometrical behavior of the robot has to be performed as well in position as in orientation over the global work volume. In this case, the six driven joint coordinates would be the inputs of the FL model, and the outputs would be the six components of the elastic displacement vector. The number of rules will be increased and thus the model will lose its 
interpretability. To overcome this problem, the use of a fuzzy decision tree would limit the number of rules and thus enhance the interpretability [58, 59, 40]. Another solution could be to use a gray box approach that consists in describing some parts of the elastic behavior of the robot structure by using some parametric sub-models and to link them through fuzzy logic inference systems.

\section{Application to forming process}

The elasto-geometrical modeling procedure described in the previous sections can be applied for machining, assembly or forming applications using an industrial robot. The main problem, in a forming context, concern the TCP pose errors due to the robot's elastic deformations induced by the exerted quasi-static forces during the process. This leads to deviations of the tool from the target path. To enhance the robot pose accuracy during the process, a compensation method is developed based on the modeling approach previously described.

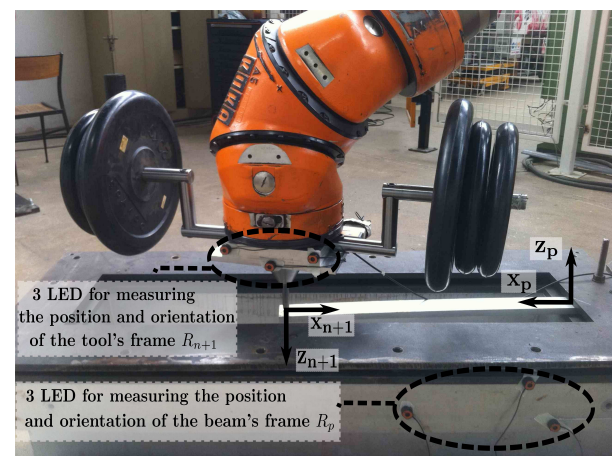

Figure 16: Experimental setup with the KUKA-IR663, a cantilever beam and its clamping device

The performance of the proposed elasto-geometrical FL model is verified through a simple but robust experimental procedure emulating a forming process. The KUKA-IR663 robot is used to bend elastically an aluminium (Al-5005) cantilever beam $(585 \times 50 \times 14 \mathrm{~mm})$ clamped on a frame as shown on Fig. 16 . The beam is deformed only elastically since its reduces the force applied by the robot and avoids a time consuming calculation of the forming force, with a FE software [7]. Here to produce the elastic deformation of the beam, the forming tool tip is moved along a trajectory defined in frame $R_{p}$ (Fig. 1). A vertical motion of $25 \mathrm{~mm}$ along $\mathbf{z}_{p}$ is planed followed by an horizontal motion of 200 $m m$ along $\mathbf{x}_{p}$ starting at the beam's free-end. During the process emulation, the actual tool path is measured by the K600-10 system. A dead weight of 920 $N$ is carried by the end-effector in order to increase the vertical payload (Fig. $16)$.

Despite its simplicity, this experimental test is realistic regarding the modeling of a forming process. The forming forces acting on the tool lead to large TCP elastic deviations (up to $1.5 \mathrm{~mm}$ ) that requires a model-based compensation. This compensation method allows to predict the path deviations due to the robot structure compliance and to plan a corrected tool path [7]. The strain 
and deflection characteristics of the cantilever beam are calculated using two approaches. Euler-Bernoulli theory is first used assuming small deflections of the beam. Then incremental analysis with reactualization of the beam geometry is involved assuming the loadings create large displacements and/or rotations. The simulation of the robot elasto-geometrical behavior along the forming path is then done using the proposed Fuzzy Logic approach. The two beam deformation models and the Fuzzy Logic model are coupled to get a robust calculation of the tool path without compensation (Fig. 17). Once this path is identified, the trajectory is corrected, computing fake poses with the FL model, so as to reach the target path.

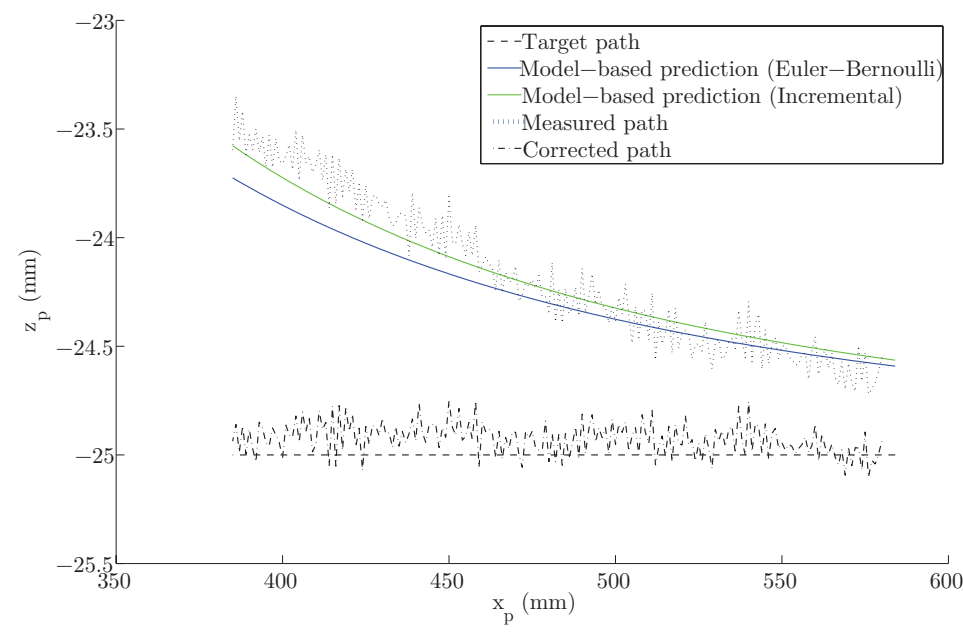

Figure 17: Target tool path, measured and simulated paths in the vertical x-z-plane of the beam frame

A significant deviation between the target and the measured path can be identified. It ranges from $0.4 \mathrm{~mm}$ to $1.5 \mathrm{~mm}$ along $\mathbf{z}_{p}$ direction. Obviously, the nearer the tool is from the free-end of the beam, the lower the vertical forming force is. The comparison between the measured and simulated paths shows good qualitative and quantitative agreements. As one can see, the model based path prediction using the incremental analysis leads to more accurate results. Both horizontal and vertical forces induced by the beam deformation are considered and thus the calculation of the robot TCP elastic displacements is more accurate. The gap between the predicted trajectories respectively obtained with EulerBernoulli and incremental approaches is about $0.2 \mathrm{~mm}$ when the tool is near the beam's embedment. The robot trajectory is corrected efficiently using the incremental model-based prediction. Fig. 17 shows the compensated tool path and the target path. The tool path accuracy has been enhanced from $80 \%$ applying the presented compensation strategy based on the elasto-geometrical modeling of the robot structure using the FL model. The variation remaining between the target path and the compensated tool path can be explained partly by not taking into account the longitudinal force resulting from the friction of the tool on the beam's surface. These results validate the modeling approach proposed in this paper. 


\section{Conclusion}

This paper studies the elasto-geometrical calibration of robot manipulators in order to improve their static pose accuracy and to fit with machining, forming or assembly process precision requirements. A key result is that the proposed framework can be used to obtain parametric or fuzzy logic elasto-geometrical models of robot structures. The first solution consists in using analytical finite element theory for the calculation of the equivalent structure stiffness matrix required to predict accurately the TCP pose errors under high level forces. The Forward Elasto-Geometrical model of the KUKA-IR663 is derived analytically in a systematic manner and its complete identification is then performed using experimental data obtained with the NIKON METROLOGY K600-10 measurement system. The errors on the geometrical parameters and the stiffness parameters are identified. The simulation results obtained with this model have been compared to experimental results in order to show the efficiency of the parametric approach. However, while considering the whole work volume, the measured elastic displacements exhibit nonlinear behavior that cannot be described precisely using conventional parametric method.

Therefore, a second modeling approach, based on a fuzzy inference system, is proposed. In fuzzy logic modeling, the model development is mainly driven by measurement data and has been proved to have the ability of modeling elastic behavior without any priori assumptions. The fuzzy approach can be considered to be a more versatile and have an efficient mathematical structure for nonlinear system mapping. The proposed procedure has been successfully validated by experimental investigations. The experimental results show the method relevance to minimize defects due to the compliances of the robot's structure. The TCP's pose accuracy has been improved up to $\pm 0.15 \mathrm{~mm}$ for the elastic bending of an aluminium cantilever beam and this relative value of the maximal error fits the tolerance level of incremental forming applications.

\section{Acknowledgement}

The authors would like to thank EngineSoft and Esteco for providing access to modeFRONTIER. The authors also wish to acknowledge the support of Nikon Metrology, especially M. Loïc MARQUET.

\section{Appendix A. Four-bar linkage}

For the calculation of the relation $U$, the assumption that the four bar linkage is a plane mechanism is made (Fig. A.18). In other words, the axes of the joints 2, 3, 8, 9 and 10 are assumed to be perfectly parallel. Using the closure equation of the linkage, ${ }^{11} \mathbf{T}_{9}{ }^{9} \mathbf{T}_{8}{ }^{8} \mathbf{T}_{1}{ }^{1} \mathbf{T}_{2}{ }^{2} \mathbf{T}_{3}{ }^{3} \mathbf{T}_{10}=\mathbf{I}_{4}$, the value of $\theta_{3}^{k}$ is calculated as a function of the other loop parameters as follows:

$$
\begin{aligned}
& \theta_{3}^{k}=\operatorname{Atan} 2\left(\sin \left(\theta_{3}^{k}\right), \cos \left(\theta_{3}^{k}\right)\right) \\
& =U\left(\theta_{2}^{k}, \theta_{8}^{k}, d_{3}, d_{9}, d_{10}, d_{11}\right) \\
& \text { where: } \quad \sin \left(\theta_{3}\right)=\frac{B_{1} B_{3}-B_{2} \sqrt{B_{1}^{2}+B_{2}^{2}-B_{3}^{2}}}{B_{1}^{2}+B_{2}^{2}} \text {, } \\
& \cos \left(\theta_{3}\right)=\frac{B_{2} B_{3}-B_{1} \sqrt{B_{1}^{2}+B_{2}^{2}-B_{3}^{2}}}{B_{1}^{2}+B_{2}^{2}},
\end{aligned}
$$


and:
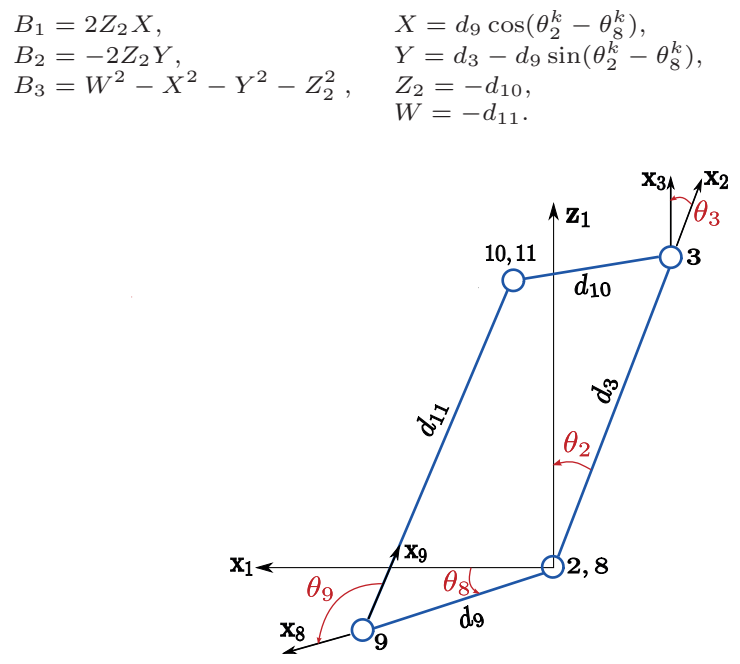

Figure A.18: Description of the four-bar linkages

The relation $V$ linking the actuated translational displacements ${r_{2}^{\prime}}^{k}$ to the joint values $\theta_{2}^{k}$ and the parameters of the screw is calculated considering the resulting slider crank type mechanism of Fig. A.19.

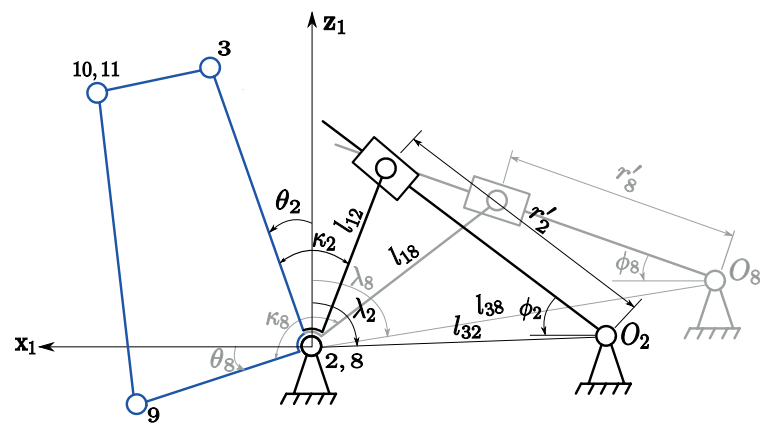

Figure A.19: Ball screws for the actuation of joints 2 and 8

Writing the closure equation of the mechanism in the plane, the following relation is obtained:

$$
\theta_{2}^{k}=V\left(r_{2}^{\prime k}, \kappa_{2}, \lambda_{2}, l_{12}, l_{32}\right)
$$

where: $\quad \theta_{2}^{k}=\kappa_{2}-\operatorname{Atan} 2\left(\sin \left(\theta_{2}^{k}\right), \cos \left(\theta_{2}^{k}\right)\right)+\frac{\pi}{2}$,

$$
\begin{aligned}
& \sin \left(\theta_{2}^{k}\right)=\frac{X_{2} Z_{2}-Y_{2} \sqrt{X_{2}^{2}+Y_{2}^{2}-Z_{2}^{2}}}{X_{2}^{2}+Y_{2}^{2}}, \\
& \cos \left(\theta_{2}^{k}\right)=\frac{Y_{2} Z_{2}-X_{2} \sqrt{X_{2}^{2}+Y_{2}^{2}-Z_{2}^{2}}}{X_{2}^{2}+Y_{2}^{2}},
\end{aligned}
$$

and: $\quad X_{2}=2 y_{B} l_{12}, \quad x_{B}=l_{32} \cos \left(\frac{\pi}{2}-\lambda_{2}\right)$,

$$
\begin{aligned}
& Y_{2}=2 x_{B} l_{12}, \\
& Z_{2}=r_{2}^{\prime k}-x_{B}^{2}-y_{B}^{2}-l_{12}^{2} .
\end{aligned}
$$

In the same way, the slider crank mechanism that drives the joint 8 is considered to calculate the function $W$ linking $\theta_{8}^{k}$ to $r_{8}^{\prime k}$ and the ball screw parameters:

$$
\theta_{8}^{k}=W\left(r_{8}^{\prime k}, \kappa_{8}, \lambda_{8}, l_{18}, l_{38}\right)
$$




\section{Appendix B. Columns of ${ }^{0} \mathbf{J}_{E}^{\prime k}$}

The submatrices involved in the calculation of ${ }^{0} \mathbf{J}_{E}^{\prime k}$ are obtained using the following relations [54] :

- ${ }^{0} \mathbf{J}_{1, E}^{\prime k}=\left[\begin{array}{llll}{ }^{0} \mathbf{J}_{\alpha_{1, E}}^{k} & { }^{0} \mathbf{J}_{d_{1, E}}^{k} & { }^{0} \mathbf{J}_{\theta_{1, E}}^{k} & { }^{0} \mathbf{J}_{r_{1, E}}^{k}\end{array}\right]$

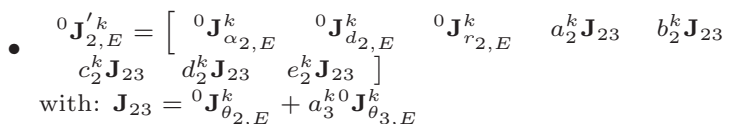

- ${ }^{0} \mathbf{J}_{3, E}^{\prime k}=\left[\begin{array}{llll}{ }^{0} \mathbf{J}_{\beta_{3, E}}^{k} & { }^{0} \mathbf{J}_{\alpha_{3, E}}^{k} & { }^{0} \mathbf{J}_{d_{3, E}}^{k}+b_{3}^{k 0} \mathbf{J}_{\theta_{3, E}}^{k} & { }^{0} \mathbf{J}_{r_{3, E}}^{k}\end{array}\right]$

- ${ }^{0} \mathbf{J}_{4, E}^{\prime k}=\left[\begin{array}{llll}{ }^{0} \mathbf{J}_{\alpha_{4, E}}^{k} & { }^{0} \mathbf{J}_{d_{4, E}}^{k} & { }^{0} \mathbf{J}_{\theta_{4, E}}^{k} & { }^{0} \mathbf{J}_{r_{4, E}}^{k}\end{array}\right]$

- ${ }^{0} \mathbf{J}_{5, E}^{\prime k}=\left[\begin{array}{llll}{ }^{0} \mathbf{J}_{\alpha_{5, E}}^{k} & { }^{0} \mathbf{J}_{d_{5, E}}^{k} & \left({ }^{0} \mathbf{J}_{\theta_{5, E}}^{k}-{ }^{0} \mathbf{J}_{\theta_{6, E}}^{k}\right) & { }^{0} \mathbf{J}_{r_{5, E}}^{k}\end{array}\right]$

- ${ }^{0} \mathbf{J}_{6, E}^{\prime k}=\left[\begin{array}{lll}{ }^{0} \mathbf{J}_{\alpha_{6, E}}^{k} & { }^{0} \mathbf{J}_{d_{6, E}}^{k} & { }^{0} \mathbf{J}_{r_{6, E}}^{k}\end{array}\right]$

- ${ }^{0} \mathbf{J}_{7, E}^{\prime k}=\left[\begin{array}{llll}{ }^{0} \mathbf{J}_{\alpha_{7, E}}^{k} & { }^{0} \mathbf{J}_{d_{7, E}}^{k} & { }^{0} \mathbf{J}_{\theta_{7, E}}^{k} & { }^{0} \mathbf{J}_{r_{7, E}}^{k}\end{array}\right]$

- ${ }^{0} \mathbf{J}_{E, E}^{\prime k}=\left[\begin{array}{llll}{ }^{0} \mathbf{J}_{\beta_{E, E}}^{k} & { }^{0} \mathbf{J}_{\alpha_{E, E}}^{k} & { }^{0} \mathbf{J}_{d_{E, E}}^{k} & { }^{0} \mathbf{J}_{r_{E, E}}^{k}\end{array}\right]$

${ }^{0} \mathbf{J}_{8, E}^{\prime k}=\left[\begin{array}{lll}-a_{8}^{k} a_{3}^{k 0} \mathbf{J}_{\theta_{3, E}}^{k} & -b_{8}^{k} a_{3}^{k 0} \mathbf{J}_{\theta_{3, E}}^{k} & -c_{8}^{k} a_{3}^{k 0} \mathbf{J}_{\theta_{3, E}}^{k}\end{array}\right.$

- $\left.\quad-d_{8}^{k} a_{3}^{k 0} \mathbf{J}_{\theta_{3, E}}^{k} \quad-e_{8}^{k} a_{3}^{k 0} \mathbf{J}_{\theta_{3, E}^{k}}^{k}\right]$

- ${ }^{0} \mathbf{J}_{9, E}^{\prime k}=\left[c_{3}^{k 0} \mathbf{J}_{\theta_{3, E}^{k}}^{k}\right]$

- ${ }^{0} \mathbf{J}_{10, E}^{\prime k}=\left[d_{3}^{k 0} \mathbf{J}_{\theta_{3, E}}^{k}\right]$

- ${ }^{0} \mathbf{J}_{11, E}^{\prime k}=\left[e_{3}^{k 0} \mathbf{J}_{\theta_{3, E}^{k}}^{k}\right]$

\section{Appendix C. Geometrical calibration}

\section{7 poses used for measurements}

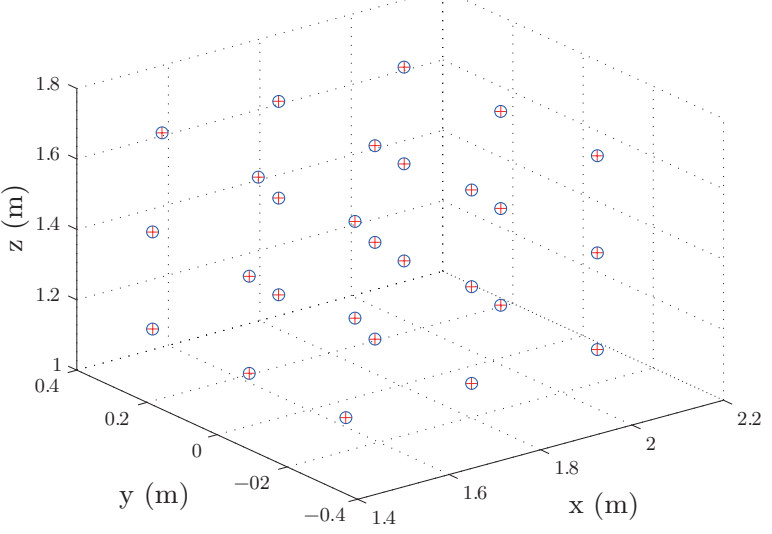

Figure C.20: Robot poses used for measurements 
Linear combinations between the parameters of $d \xi^{B}$ and the parameters that cannot be identified independently $d \kappa_{8}, d \kappa_{2}, d r^{\prime}{ }_{8}, d d_{9}, d r_{2}, d r_{7}$ :

$$
\left\{\begin{array}{l}
d r_{2}^{\prime i d}=d r_{2}^{\prime}+0.003 . d d_{9}-0.011 . d r_{8}^{\prime} \\
d \lambda_{2}^{i d}=d \lambda_{2}-d \kappa_{2} \\
d l_{12}^{i d}=d l_{12}+0.0011 . d d_{9}+0.0066 d r_{8}^{\prime} \\
d l_{32}^{i d}=d l_{32}+0.0019 . d d_{9}-0.018 . d r_{8}^{\prime} \\
d \lambda_{8}^{i d}=d \lambda_{8}-d \kappa_{8}+0.098 . d d_{9}+-0.78 . d r_{8}^{\prime} \\
d l_{18}^{i d}=d l_{18}-0.0014 . d r_{8}^{\prime} \\
d l_{38}^{i d}=d l_{38}-0.0012 . d d_{9}-0.84 . d r_{8}^{\prime} \\
d r_{1}^{i d}=d r_{1}-0.0012 . d d_{9} \\
d d_{3}^{i d}=d d_{3}+0.0013 . d d_{9} \\
d r_{3}^{i d}=d r_{3}+d r_{2} \\
d d_{5}^{i d}=d d_{5}+0.0053 . d d_{9}+0.0025 . d r_{8}^{\prime} \\
d r_{5}^{i d}=d r_{5}-0.0036 . d d_{9}-0.0019 . d r_{8}^{\prime} \\
d r_{6}^{i d}=d r_{6}-0.0038 . d d_{9}-0.0021 . d r_{8}^{\prime} \\
d d_{7}^{i d}=d d_{7}-0.0057 . d d_{9}-0.0025 . d r_{8}^{\prime} \\
d d_{10}^{i d}=d d_{10}-0.82 . d d_{9} \\
d d_{11}^{i d}=d d_{11}-0.39 . d d_{9} \\
d r_{E}^{i d}=d r_{E}+d r_{7}
\end{array} .\right.
$$

Values of identified geometrical errors (deg, mm, $\times$ : non identifiable):

$$
\begin{aligned}
& \left\{\begin{array}{l}
d \alpha_{1}^{i d}=-0.0045 \\
d d_{1}=-0.1117 \\
d \theta_{1}^{i d}=0.0059 \\
d r_{1}^{i d}=-0.04512
\end{array}\right. \\
& \left\{\begin{array}{l}
d \alpha_{2}^{i d}=-0.0058 \\
d d_{2}^{i d}=0.0944 \\
d r_{2}^{\prime i d}=-0.0453 \\
d \kappa_{2}^{i d}=\times \\
d \lambda_{2}^{i d}=-0.0026 \\
d l_{12}^{i d}=-0.0456 \\
d l_{32}^{i d}=\times \\
d r_{2}^{i d}=\times
\end{array}\right. \\
& \left\{\begin{array}{l}
d \beta_{3}^{i d}=0.0067 \\
d \alpha_{3}^{i d}=-0.0013 \\
d d_{3}^{i d}=0.1804 \\
d r_{3}^{i d}=-0.0855
\end{array}\right. \\
& \left\{\begin{array}{l}
d \alpha_{4}^{i d}=0.0032 \\
d d_{4}^{i d}=-0.4196 \\
d \theta_{4}^{i d}=0.0395 \\
d r_{4}^{i d}=-0.030
\end{array}\right. \\
& \left\{\begin{array}{l}
d \alpha_{5}^{i d}=-0.0033 \\
d d_{5}^{i d}=0.0729 \\
d \theta_{5}^{i d}=-0.0056 \\
d r_{5}^{i d}=-0.0995
\end{array}\right.
\end{aligned}
$$




$$
\begin{gathered}
\left\{\begin{array}{l}
d \alpha_{6}^{i d}=-0.0011 \\
d d_{6}^{i d}=-0.4267 \\
d r_{6}^{i d}=-0.1536
\end{array}\right. \\
\left\{\begin{array}{l}
d \alpha_{7}^{i d}=0.0272 \\
d d_{7}^{d i}=-0.6448 \\
d \theta_{7}^{i d}=0.0228 \\
d r_{7}^{i d}=\times
\end{array}\right. \\
\left\{\begin{array}{l}
d \beta_{E}^{i d}=0.0132 \\
d \alpha_{E}^{i d}=0.0048 \\
d d_{E}^{i d}=-0.0285 \\
d r_{E}^{i d}=0.7799
\end{array}\right. \\
\left\{\begin{array}{l}
d r_{8}^{\prime i d}=\times \\
d \kappa_{8}^{i d}=\times \\
d \lambda_{8}^{i d}=0.0027 \\
d l_{18}^{i d}=0.3134 \\
d l_{38}^{i d}=-0.080
\end{array}\right. \\
\left\{\begin{array}{l}
d d_{9}^{i d}=\times \\
d d_{10}^{i d}=-0.4441 \\
d d_{11}^{i d}=0.2074
\end{array}\right.
\end{gathered}
$$

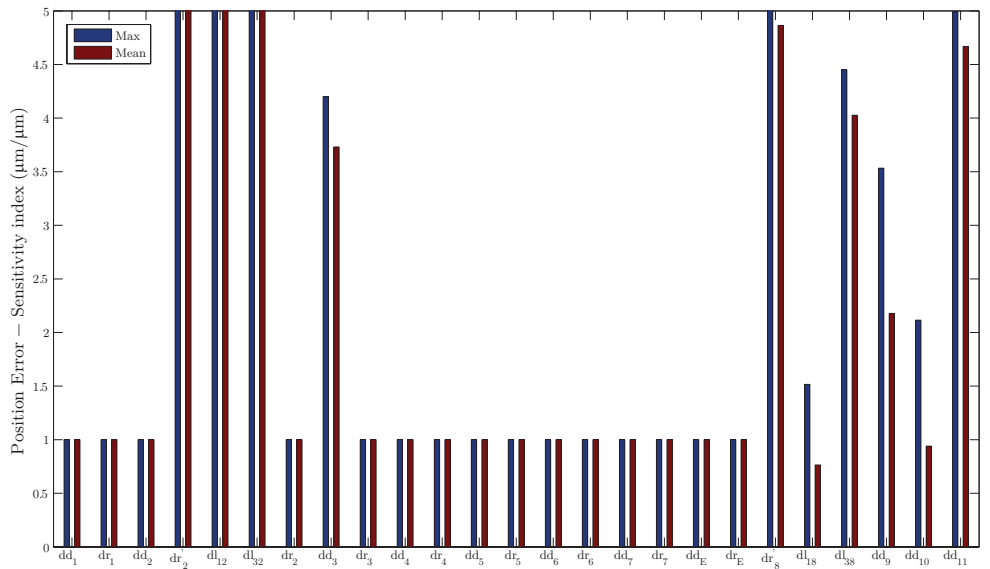

Figure C.21: Sensitivity of the end-effector position to the length geometrical errors 


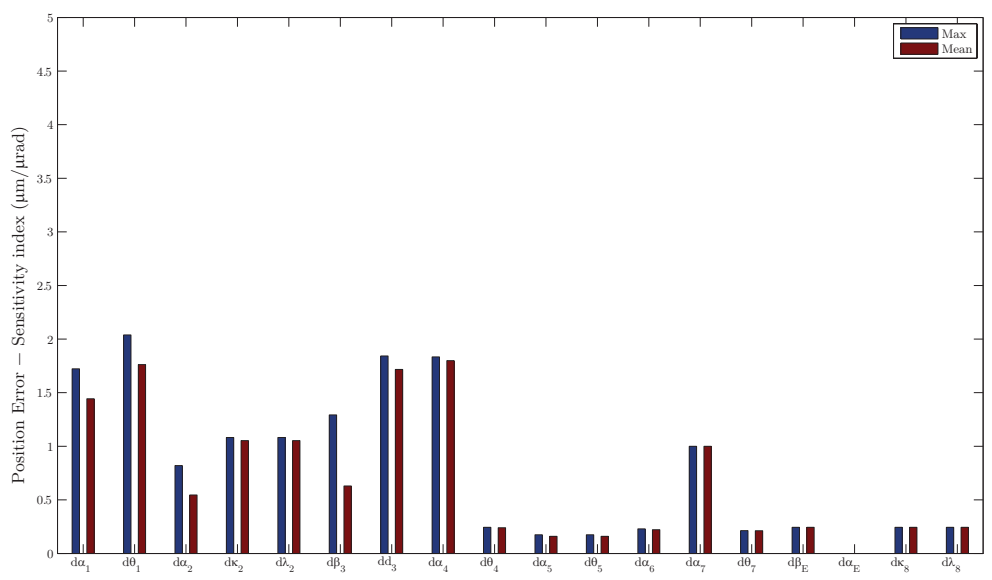

Figure C.22: Sensitivity of the end-effector position to the angular geometrical errors

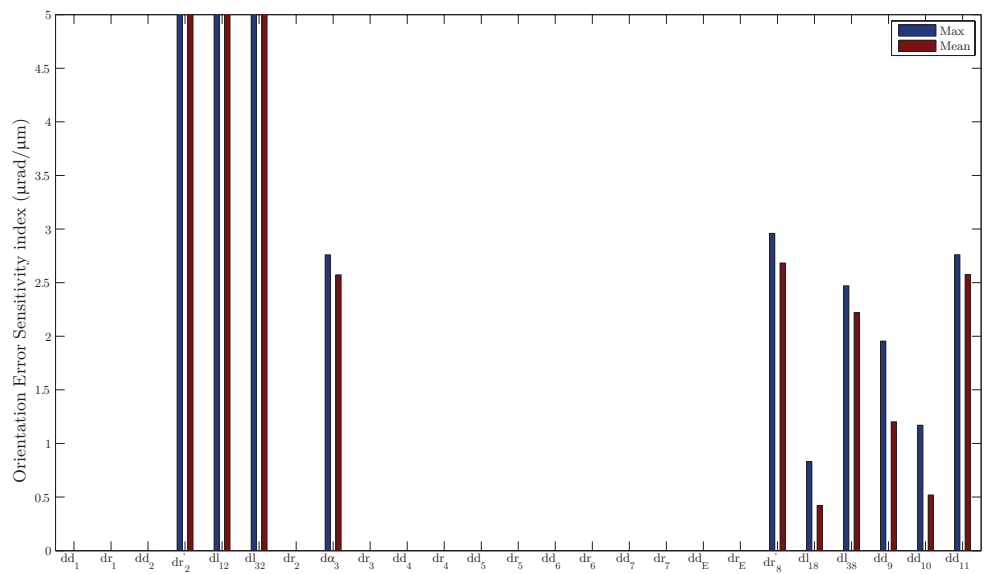

Figure C.23: Sensitivity of the end-effector orientation to the length geometrical errors 


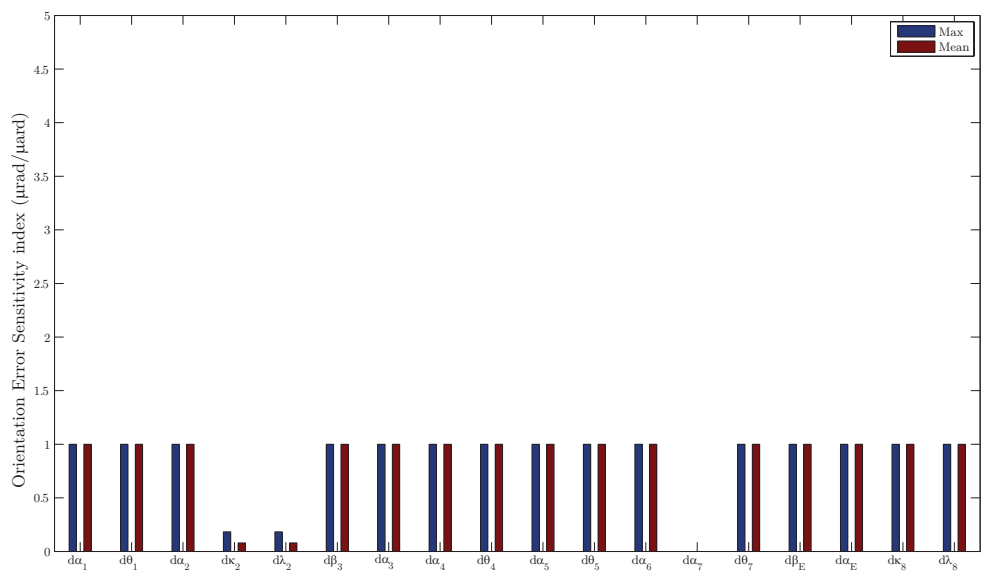

Figure C.24: Sensitivity of the end-effector orientation to the length geometrical errors

Appendix D. Measured elastic displacements of the end-point of the KUKA-IR663
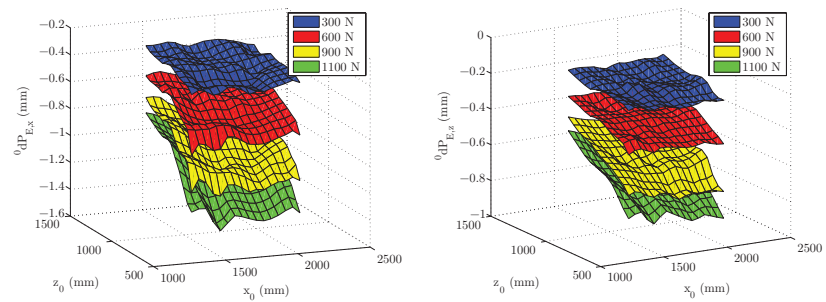

Figure D.25: Horizontal and vertical measured elastic displacements of the end-point for horizontal static loads of $300,600,900$ and $1100 \mathrm{~N}$
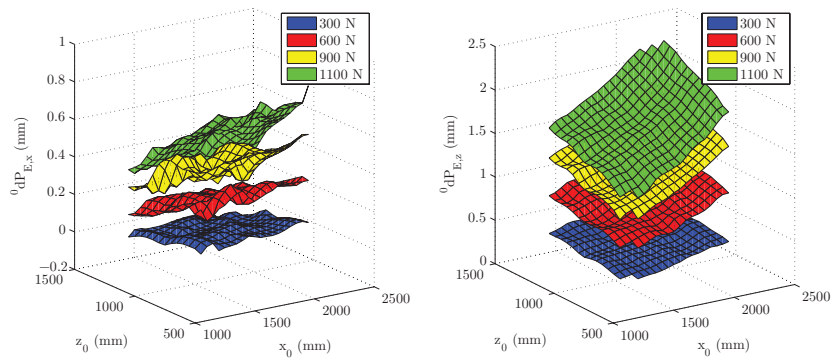

Figure D.26: Horizontal and vertical measured elastic displacements of the end-point for vertical static loads of $300,600,900$ and $1100 \mathrm{~N}$ 


\section{References}

[1] H. Meier, B. Buff, R. Laurischkat, V. Smukala, Increasing the part accuracy in dieless robot-based incremental sheet metal forming, CIRP Annals Manufacturing Technology (2009) 58 233-238.

[2] O. Marcotte, L. Vanden-Abeele, 2D and 3D Friction Stir Welding with articulated robot arm, in: The 8th Int. Friction Stir Welding Symposium, Timmendorfer Strand, Germany, 2010, pp. 1-20.

[3] H. Nakamura, T. Itaya, K. Yamamoto, T. Koyama, Robot autonomous error calibration method for off line programming system, in: The IEEE Int. Conf. on Robotics \& Automation, Vol. 2, Nagoya, Japon, 1995, pp. $1775-1783$.

[4] K. Schroer, S. L. Albright, A. Lisounkin, Modeling closed-loop mechanisms in robots for purposes of calibration, IEEE Trans. on Robotics and Automation 13 (2) (1997) 218-229.

[5] G. Alici, B. Shirinzadeh, Enhanced stiffness modeling, identification and characterization for robot manipulators, IEEE Trans. on Robotics and Automation 21 (4) (2005) 554-564.

[6] S. Marie, P. Maurine, Elasto-geometrical modelling of closed-loop industrial robots used for machining applications, in: The IEEE Int. Conf. on Robotics and Automation, 2008, pp. 1294-1300.

[7] J. Belchior, M. Guillo, E. Courteille, P. Maurine, L. Leotoing, D. Guines, Off-line compensation of the tool path deviations on robotic machining: Application to incremental sheet forming, Robotics and ComputerIntegrated Manufacturing, (2012).

[8] J. Denavit, R. S. Hartenberg, A kinematic notation for a lower-pair mechanisms based on matrices, ASME Journal of Applied Mechanics 22 (2) (1955) 215-221.

[9] S. Hayati, Robot arm geometrical link parameter estimation, in: The 22nd Int. IEEE Conf. on Decision and Control, San Antonio, Texas, USA, 1983, pp. 1477-1483.

[10] W. Khalil, J.-F. Kleinfinger, A new geometric notation for open and closed loop robots, in: The IEEE Int. Conf. on Robotics and Automation, Vol. 2, San Francisco, Californy, USA, 1986, pp. 1174-1180.

[11] J. M. Hollerbach, A survey of kinematic calibration, Robotics Review (1989) 227-242.

[12] G. Alici, B. Shirinzadeh, Laser interferometry based robot position error modelling for kinematic calibration, IEEE Trans. on Robotics and Automation 21 (4) (2003) 3588-3593.

[13] A. Nubiola, I. A. Bonev, Absolute calibration of an ABB IRB 1600 robot using a laser tracker, Robotics and Computer-Integrated Manufacturing 29 (1) (2013) 236-245. 
[14] W. Khalil, M. Gautier, C. Enguehard, Identifiable parameters and optimum configurations for robot calibration, Robotica 9 (1991) 63-70.

[15] M. W. Spong, K. Khorasani, P. V. Kokotovic, An integral manifold approach to the feedback control of flexible joint robots, IEEE Journal of Robotics and Automation 3 (4) (1987) 291-300.

[16] W. Khalil, M. Gautier, Modeling of mechanical systems with lumped elasticity, in: The IEEE Int. Conf. on Robotics \& Automation, San Francisco, USA, 2000, pp. 3964-3969.

[17] A. Bres, B. Monsarrat, L. Dubourg, L. Birglen, C. Perron, M. Jahazi, L. Baron, Simulation of Robotic Friction Stir Welding of Aerospace Components, Int. Journal of Industrial Robots 37 (1) (2010) 36-50.

[18] M. Gautier, W. Khalil, Direct calculation of minimum set of inertial parameters of serial robots, IEEE Trans. on Robotics and Automation 6 (3) (1990) 368-373.

[19] W. Khalil, E. Dombre, Modeling, identification and control of robots, Hermes Penton Ltd, 2002.

[20] C. Canudas de Wit, B. Siciliano, B. G., Theory of robot control, Springer, 1996.

[21] J. L. Caenen, J. C. Angue, Identification of geometric and nongeometric parameters of robots, in: The IEEE Int. Conf. on Robotics and Automation, 1990, pp. 1032-1037 vol.2.

[22] D. Zhang, C. Gosselin, Kinetostatic modeling of n-dof parallel mechanisms with a passive constraining leg and prismatic actuators, Journal of Mechanical Design 123 (2001) 375-381.

[23] D. Zhuang, C. Gosselin, Kinetostatic modeling of parallel mechanisms with a passive constraining leg and revolute actuators, Mechanism and Machine Theory 37 (2002) 599-617.

[24] C. Gosselin, D. Zhuang, Stiffness analysis of parallel mechanisms using a lumped model, Int. Journal of Robotics and Automation 17 (1) (2002) $17-27$.

[25] C. Huang, W.-H. Hung, I. Kao, New conservative stiffness mapping for the stewart-gough platform, in: The IEEE Int. Conf. on Robotics \& Automation, Washington, DC, USA, 2002, pp. 823-828.

[26] O. Company, F. Pierrot, J.-C. Fauroux, A method for modeling analytical stiffness of a lower mobility parallel manipulator, in: The IEEE Int. Conf. on Robotics and Automation, Barcelone, Espagne, 2005, pp. 3232-3237.

[27] F. Majou, C. Gosselin, P. Wenger, D. Chablat, Parametric stiffness analysis of the orthoglide, Mechanism and Machine Theory 42 (2007) 296-311.

[28] C. Dumas, S. Caro, M. Cherif, S. Garnier, B. Furet, Joint stiffness identification of industrial serial robots 30 (4) (2012) 649-659. 
[29] A. Klimchik, A. Pashkevich, Y. Wu, S. Caro, B. Furet, Design of calibration experiments for identification of manipulator elastostatic parametersJournal of Mechanics Engineering and Automation 2 (2012) 531-542.

[30] F. Boyer, W. Khalil, Kinematic model of a multi-beam structure undergoing large elastic displacements and rotations. part one: model of an isolated beam, Mechanism and Machine Theory 34 (1999) 205-222.

[31] D. Deblaise, P. Maurine, Effective geometrical calibration of a delta parallel robot used in neurosurgery, in: The IEEE Int. Conf. on Intelligent Robots and Systems, Edmonton, Alberta, Canada, 2005, pp. 1313-1318.

[32] D. Deblaise, X. Hernot, P. Maurine, A systematic analytical method for pkm stiffness matrix calculation, in: The IEEE Int. Conf. on Robotics and Automation, Orlando, Floride, USA, 2006, pp. 4213-4219.

[33] C. Clinton, G. Zhang, A. Wavering, Stiffness modeling of a stewartplatform-based miling machine, Tech. rep., Department of Mechanical Enginering and Institute for Systems Research, University of Maryland (1997).

[34] B. C. Bouzgarrou, J. Fauroux, G. Gogu, Y. Heerah, Rigidity analysis of T3R1 parallel robot with uncoupled kinematics, in: The 35th Int. Symposium on Robotics (ISR 2004), Paris, France, 2004.

[35] C. Corradini, J. Fauroux, K. S., O. Company, Evaluation of a 4 degree of freedom parallel manipulator stiffness, in: The 11th World Congress in Mechanism and Machine Science, IFToMM, Tianjin, Chine, 2004.

[36] M. Sugeno, K. Tanaka, Successive identification of a fuzzy model and its applications to prediction of a complex system, Fuzzy Sets and systems 42 (3) (1991) 315-334.

[37] M. Sugeno, T. Yasukawa, A fuzzy-logic-based approach to qualitative modeling, Fuzzy Systems, IEEE Transactions on 1 (1) (1993) 7-31.

[38] R. Babuska, H. Verbruggen, An overview of fuzzy modeling for control, Control Engineering Practice 4-11 (1996) 1593-1606.

[39] A. Gaweda, J. Zurada, Data-driven linguistic modeling using relational fuzzy rules, Fuzzy Systems, IEEE Transactions on 11 (1) (2003) 121-134.

[40] S. M. Zhou, J. Q. Gan, Low-level interpretability and high-level interpretability: a unified view of data-driven interpretable fuzzy system modelling, Fuzzy Sets and Systems 159 (23) (2008) 3091-3131.

[41] W. Khalil, M. Gautier, Calculation of the identifiable parameters for robot calibration, in: The 9th IFAC/IFORS Symposium on Identification and System Parameter Estimation, Budapest, Hongrie, 1991, pp. 888-892.

[42] W. Khalil, S. Besnard, Geometric calibration of robots with flexible joints and links, Journal of Intelligent and Robotic Systems 34 (2002) 357-379.

[43] W.-K. Yoon, T. Suehiro, Y. Tsumaki, M. Uchiyama, Stiffness analysis and design of a compact modified delta parallel mechanism, Robotica 22 (4) (2004) 463-475. 
[44] G. Ecorchard, Static accuracy enhancement of redundantly actuated parallel kinematic machine toos, Ph.D. thesis, Institut National des Sciences Appliquées de Rennes-France, Chemnitz University of Technology-Germany.

[45] T. Takagi, M. Sugeno, Fuzzy identification of system and its applications to modelling and control, IEEE Trans. on Fuzzy Systems, Man and Cybernetics 15-1 (1985) 116-130.

[46] J. Castro, Fuzzy logic controllers are universal approximators, IEEE Trans. on Systems, Man, and Cybernetics 25-4 (1995) 629-635.

[47] B. Kosko, Fuzzy systems as universal approximators, Computers, IEEE Transactions on 43 (11) (1994) 1329-1333.

[48] L. Zadeh, Fuzzy algorithms, Information and Control 12 (1968) 94-102.

[49] P. Glorennec, Algorithmes d'apprentissage pour systèmes d'inférence floue, no. ISBN 2-7462-0044-9, 1999.

[50] F. Benmakrouha, Parameter identification in a fuzzy system with insufficient data, in: Proceedings of the Sixth IEEE Int. Conf. on Fuzzy Systems, Vol. 1, 1986, pp. 537-542.

[51] F. Solis, J. Wets, Minimization by random search techniques, Mathematics of Operation Research 6.

[52] C. Raymond, Commande floue monovariable et multivariable selon l'expertise et par modélisation floue, Ph.D. thesis, ANRT (1995).

[53] W. Khalil, J. F. Kleinfinger, Minimum operations and minimum parameters of the dynamic models of tree structure robots, IEEE Journal of Robotics and Automation 3 (6) (1987) 517-526.

[54] W. Khalil, J. Caenen, C. Enguehard, Identification and calibration of robot, in: First Int. Symposium on Experimental Robot, Montreal, Canada, 1989.

[55] S. Caro, P. Wenger, B. Fouad, D. Chablat, Sensitivity analysis of the orthoglide: a three-dof translational parallel kinematic machine, Transaction of the ASME 128 (2006) 392-402.

[56] G. Ecorchard, R. Neugebauer, P. Maurine, Elasto-geometrical modeling and calibration of redundantly actuated pkms, Mechanism and Machine Theory 45 (5) (2010) 795-810.

[57] C. M. Fonseca, P. J. Fleming, Multiobjective optimization and multiple constraint handling with evolutionary algorithms, IEEE Trans. on Systems, Man, and Cybernetics 28 (1998) 26-37.

[58] L. Breiman, Classification and regression trees, Chapman \& Hall/CRC, 1984.

[59] S. Guillaume, Designing fuzzy inference systems from data: An interpretability-oriented review, IEEE Trans. on Fuzzy Systems 9 (3) (2001) 426-443. 